\title{
Inflowing gas onto a compact obscured nucleus in Arp 299A ${ }^{\star}$ Herschel spectroscopic studies of $\mathrm{H}_{2} \mathrm{O}$ and $\mathrm{OH}$
}

\author{
N. Falstad ${ }^{1}$, E. González-Alfonso ${ }^{2}$, S. Aalto ${ }^{1}$, and J. Fischer ${ }^{3}$ \\ 1 Department of Earth and Space Sciences, Chalmers University of Technology, Onsala Space Observatory, 43992 Onsala, Sweden \\ e-mail: niklas. falstad@chalmers. se \\ 2 Universidad de Alcalá de Henares, Departamento de Física, Campus Universitario, 28871 Alcalá de Henares, Madrid, Spain \\ 3 Naval Research Laboratory, Remote Sensing Division, 4555 Overlook Ave SW, Washington, DC 20375, USA
}

Received 3 June 2016 / Accepted 2 November 2016

\begin{abstract}
Aims. We probe the physical conditions in the core of Arp 299A and try to put constraints on the nature of its nuclear power source. Methods. We used Herschel Space Observatory far-infrared and submillimeter observations of $\mathrm{H}_{2} \mathrm{O}$ and $\mathrm{OH}$ rotational lines in Arp 299A to create a multi-component model of the galaxy. In doing this, we employed a spherically symmetric radiative transfer code.

Results. Nine $\mathrm{H}_{2} \mathrm{O}$ lines in absorption and eight in emission, as well as four $\mathrm{OH}$ doublets in absorption and one in emission, are detected in Arp 299A. No lines of the ${ }^{18} \mathrm{O}$ isotopologues, which have been seen in compact obscured nuclei of other galaxies, are detected. The absorption in the ground state $\mathrm{OH}^{2} \Pi_{3 / 2}-{ }^{2} \Pi_{3 / 2} \frac{5}{2}^{+}-\frac{3}{2}^{-}$doublet at $119 \mu \mathrm{m}$ is found redshifted by $\sim 175 \mathrm{~km} \mathrm{~s}^{-1} \mathrm{compared}$ with other $\mathrm{OH}$ and $\mathrm{H}_{2} \mathrm{O}$ lines, suggesting a low excitation inflow. We find that at least two components are required in order to account for the excited molecular line spectrum. The inner component has a radius of 20-25 pc, a very high infrared surface brightness $\left(\gtrsim 3 \times 10^{13} L_{\odot} \mathrm{kpc}^{-2}\right)$, warm dust $\left(T_{\mathrm{d}}>90 \mathrm{~K}\right)$, and a large $\mathrm{H}_{2}$ column density $\left(N_{\mathrm{H}_{2}}>10^{24} \mathrm{~cm}^{-2}\right)$. The modeling also indicates high nuclear $\mathrm{H}_{2} \mathrm{O}\left(1-5 \times 10^{-6}\right)$ and $\mathrm{OH}\left(0.5-5 \times 10^{-5}\right)$ abundances relative to $\mathrm{H}$ nuclei. The outer component is larger $(50-100 \mathrm{pc})$ with slightly cooler dust (70-90 K) and molecular abundances that are approximately one order of magnitude lower. In addition to the two components that account for the excited $\mathrm{OH}$ and $\mathrm{H}_{2} \mathrm{O}$ lines, we require a much more extended inflowing component to account for the $\mathrm{OH}^{2} \Pi_{3 / 2}-{ }^{2} \Pi_{3 / 2} \frac{5}{2}^{+}-\frac{3}{2}^{-}$doublet at $119 \mu \mathrm{m}$.

Conclusions. The Compton-thick nature of the core makes it difficult to determine the nature of the buried power source, but the high surface brightness indicates that it is an active galactic nucleus and/or a dense nuclear starburst. Our results are consistent with a composite source. The high $\mathrm{OH} / \mathrm{H}_{2} \mathrm{O}$ ratio in the nucleus indicates that ion-neutral chemistry induced by X-rays or cosmic-rays is important. Finally we find a lower limit to the ${ }^{16} \mathrm{O} /{ }^{18} \mathrm{O}$ ratio of 400 in the nuclear region, possibly indicating that the nuclear starburst is in an early evolutionary stage, or that it is fed through a molecular inflow of, at most, solar metallicity.
\end{abstract}

Key words. ISM: molecules - galaxies: ISM - galaxies: individual: Arp 299 - line: formation - infrared: galaxies submillimeter: galaxies

\section{Introduction}

Luminous infrared galaxies (LIRGs; see the review by Sanders \& Mirabel 1996) are galaxies with infrared (IR) luminosities in excess of $10^{11} L_{\odot}$, powered by active galactic nuclei (AGN) or circumnuclear starbursts. An emerging subclass of LIRGs host compact obscured nuclei (CONs) where luminosities in excess of $10^{9} L_{\odot}$ originate in compact $(d<100$ pc) dust obscured cores (e.g. Aalto et al. 2012; Costagliola \& Aalto 2010; González-Alfonso et al. 2012, hereafter G-A12; Falstad et al. 2015). The central regions of these objects are heated by a process in which the radiation is absorbed and re-emitted at progressively longer wavelengths until the dust is optically thin to its own radiation (Rolffs et al. 2011; G-A12). Due to this radiative trapping, the power source is hidden from direct observations over a broad range of wavelengths, making it hard to discern whether it is star formation or AGN activity. Determining the nature of these power sources could potentially aid our understanding of galaxy evolution greatly.

\footnotetext{
* Herschel is an ESA space observatory with science instruments provided by European-led Principal Investigator consortia and with important participation from NASA.
}

One method to identify, characterize, and indirectly study the cores of CONs is to use molecules that couple well to the IR radiation field, such as water $\left(\mathrm{H}_{2} \mathrm{O}\right)$ and hydroxyl $(\mathrm{OH})$, for example, to probe the warm dust in these regions (e.g., González-Alfonso et al. 2004, 2008; G-A12; Falstad et al. 2015). There are indeed indications that sources with a strong OH $65 \mu \mathrm{m}{ }^{2} \Pi_{3 / 2}-{ }^{2} \Pi_{3 / 2} \frac{9}{2}-\frac{7}{2}$ doublet represent the most buried stage of starburst-AGN coevolution (González-Alfonso et al. 2015). Furthermore, submillimeter (submm) $\mathrm{H}_{2} \mathrm{O}$ lines have been detected in numerous nearby (e.g., Yang et al. 2013) and high-redshift galaxies (e.g., Impellizzeri et al. 2008; Omont et al. 2011, 2013; Bradford et al. 2011; van der Werf et al. 2011). In some cases, for example in Mrk 231 (van der Werf et al. 2010; González-Alfonso et al. 2010) and $\mathrm{Zw} 049.057$ (Falstad et al. 2015), these $\mathrm{H}_{2} \mathrm{O}$ lines have fluxes comparable to those of the carbon monoxide (CO) lines.

\subsection{The interacting system Arp 299}

Arp 299 is an interacting system consisting of the two galaxies IC 694 and NGC 3690, 22" apart, whose nuclei (Arp 299A 
and Arp 299B), as well as an overlap region (Arp 299C), are strong near-IR and radio emitters due to intense star formation (Gehrz et al. 1983; Telesco et al. 1985). Based on H I observations, Nordgren et al. (1997) determined the systemic velocity of the system to be $3121 \mathrm{~km} \mathrm{~s}^{-1}$, yielding a redshift of $z=0.010411$. At a distance of $44.8 \mathrm{Mpc}$ (linear scale: 217 pc/arcsec) (Fixsen et al. 1996; Nordgren et al. 1997, assuming $\left.H_{0}=73 \mathrm{~km} \mathrm{~s}^{-1} \mathrm{Mpc}^{-1}\right)$ the IR luminosity of the system is $L_{\mathrm{IR}} \approx 6.6 \times 10^{11} L_{\odot}$ (Sanders et al. 2003) of which Arp 299A contributes approximately 40-50\% (Alonso-Herrero et al. 2000; Charmandaris et al. 2002). All three regions also exhibit bright molecular emission from hydrogen cyanide (HCN) $J=1-0$ and carbon monoxide (CO) (Sargent \& Scoville 1991; Aalto et al. 1997). The molecular gas in the compact nucleus of Arp 299A is suggested by Aalto et al. (1997) to be unusually warm and dense. The system also exhibits hydroxyl $(\mathrm{OH})$ megamaser activity (Baan 1985) from a rotating disk of size $\lesssim 1^{\prime \prime}$ in Arp 299A (Baan \& Haschick 1990). A water $\left(\mathrm{H}_{2} \mathrm{O}\right)$ megamaser in the system was reported by Henkel et al. (2005) and interferometric observations by Tarchi et al. $(2007,2011)$ showed that the emission mainly originates in Arp 299B with a second hotspot in the inner regions of Arp 299A. Using CO and $\mathrm{HCN}$ as probes, Rosenberg et al. (2014) studied molecular gas heating in the Arp 299 system and found that Arp 299A contains more warm gas than the other two regions. They further concluded that the excitation in Arp 299B and Arp 299C is consistent with heating from ultraviolet photons in photon-dominated regions (PDRs) while the excitation in Arp 299A requires an additional heating mechanism with mechanical heating being the most likely.

Based on optical, radio, and IR data, Gehrz et al. (1983) found that the emission from Arp 299, except for the radio source in Arp 299A, is consistent with starbursts at different locations. A compact condensation of molecular gas in the nucleus of Arp 299A was suggested by Sargent \& Scoville (1991) to harbor an AGN. In their X-ray measurements of the system, Della Ceca et al. (2002) found evidence for a Comptonthick AGN, but with the spatial resolution offered by BeppoSAX they could not determine its location in the system. Higher spatial resolution observations with Chandra and XMM-Newton reveal that Arp 299B is the likely home of this AGN (Zezas et al. 2003; Ballo et al. 2004). Ballo et al. (2004), however, suggested that Arp 299A might also harbor an AGN and, based on milliarcsecond radio observations, Pérez-Torres et al. (2010) concluded that there is in fact a buried low-luminosity AGN in Arp 299A. Mid-infrared observations of the system are consistent with deeply embedded star formation in the nucleus of Arp 299A and an AGN surrounded by regions of star formation in Arp 299B (Gallais et al. 2004; Alonso-Herrero et al. 2009). Subsequent high spatial resolution mid-IR observations by Alonso-Herrero et al. (2013) also show evidence of AGN activity in both nuclei, and they estimate that the AGN in Arp 299A is five times less luminous than the one in Arp 299B, corresponding to $\sim 5 \%$ of the total luminosity of Arp 299A. In their NuSTAR observations, Ptak et al. (2015) found that the hard X-ray emission $(E>10 \mathrm{keV})$ of the system is dominated by Arp 299B, with no significant emission from the position of Arp 299A. Their interpretation is that an AGN in Arp 299A must either be heavily obscured, with $N_{\mathrm{H}}>10^{24} \mathrm{~cm}^{-2}$, or have a much lower luminosity than the AGN in Arp 299B.

In this paper, we model the dusty core of the potential CON in Arp 299A using spectroscopic observations taken with the Photodetector Array Camera and Spectrometer (PACS; Poglitsch et al. 2010) and the Spectral and Photometric Imaging Receiver (SPIRE; Griffin et al. 2010) on the Herschel Space

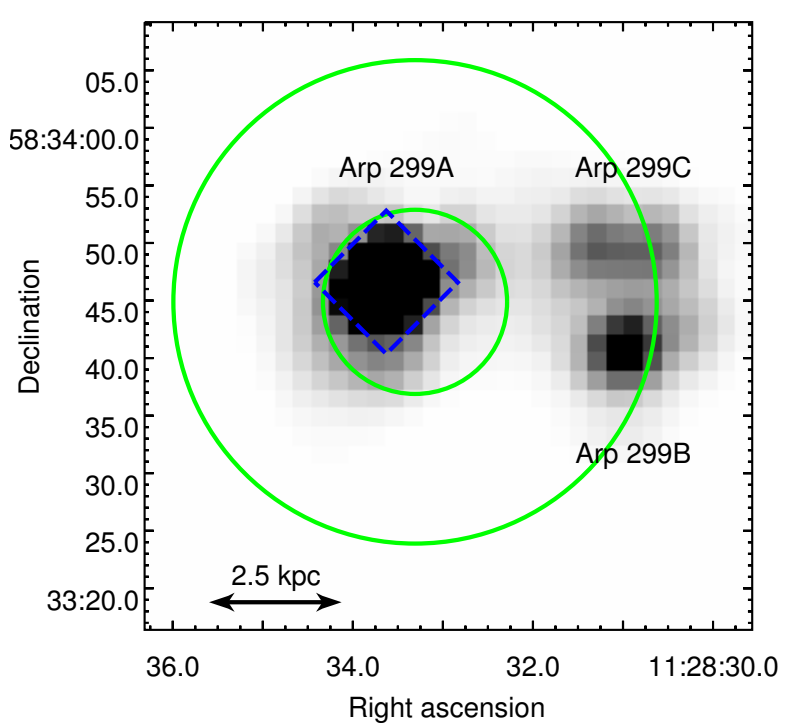

Fig. 1. SPIRE beams and the field of view of the central PACS spaxel overlaid on an archival PACS $70 \mu \mathrm{m}$ image of Arp 299. The large circle represents the largest SPIRE beam with a full-width-half-maximum (FWHM) of $\sim 42^{\prime \prime}$ at a wavelength of $\sim 670 \mu \mathrm{m}$ and the smaller circle represents the smallest beam with a FWHM of $\sim 16^{\prime \prime}$ at a wavelength of $\sim 200 \mu \mathrm{m}$. The dashed square represents the central 9.4" spaxel of the PACS spaxel array. Evidently the far-IR emission of the system is dominated by Arp 299A, which is unresolved by PACS at $70 \mu \mathrm{m}$. The coordinates are in the $\mathbf{J} 2000.0$ system.

Observatory (Pilbratt et al. 2010). The observations are described in Sect. 2, and our models are described in Sect. 3. The model results are discussed in Sect. 4, and our main conclusions are presented in Sect. 5.

\section{Observations and results}

The observations of Arp 299A were taken using the PACS and SPIRE instruments on the Herschel Space Observatory. Most of the PACS observations were conducted on October 31, 2012, as part of the Hermolirg OT2 project (PI: E. González-Alfonso). The $\mathrm{OH} 119 \mu \mathrm{m}^{2} \Pi_{3 / 2}-{ }^{2} \Pi_{3 / 2} \frac{5}{2}-\frac{3}{2}$ and $\mathrm{H}_{2} \mathrm{O} 187 \mu \mathrm{m} 4_{13} \rightarrow 4_{04}$ transitions were observed on November 21, 2011, as part of the OT1 projects OT1_shaileyd_1 (PI: S. Hailey-Dunsheath) and OT1_rmeijeri_1 (PI: R. Meijerink), respectively. All observations were performed in high spectral sampling, range spectroscopy mode. The SPIRE observation, already reported by Rosenberg et al. (2014), was conducted on June 27, 2010, as part of the OT key program Hercules (PI: P.P. van der Werf) with a single pointing centered on Arp 299A. The observation was taken in high spectral resolution, sparse image sampling mode with a resolution of $1.2 \mathrm{GHz}$ in both observing bands (447-989 GHz and 958-1545 GHz). A total of 35 repetitions (70 FTS scans) were performed, resulting in a total on-source integration time of $4662 \mathrm{~s}$. The SPIRE beams and the field of view of the central PACS spaxel, overlaid on a PACS $70 \mu \mathrm{m}$ archival image, are shown in Fig. 1. The PACS observations were well pointed while the SPIRE observation was mispointed by $\sim 3^{\prime \prime}$. The flux loss in the short wavelength array, the detector with the smallest beam, should be less than $10 \%$ due to this mispointing (Valtchanov et al. 2014).

A summary of all observations, with observation identification numbers (IDs), total durations, and the observed wavelength ranges, is provided in Table 1. All detected transitions are indicated in the energy diagram in Fig. 2. A combination 


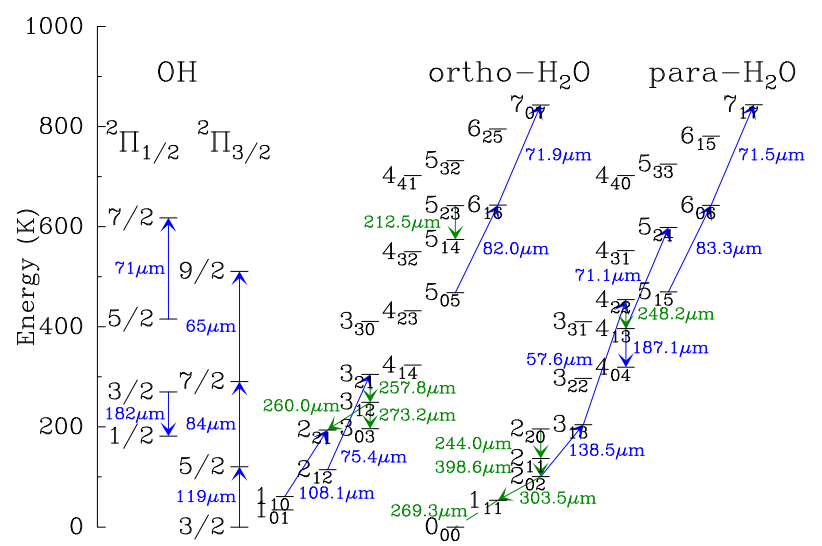

Fig. 2. Energy diagram for $\mathrm{H}_{2} \mathrm{O}$ and $\mathrm{OH}$. Transitions detected with PACS and SPIRE are indicated with solid blue and green arrows, respectively. The undetected $\mathrm{H}_{2} \mathrm{O} 269 \mu \mathrm{m} 1_{11} \rightarrow 0_{00}$ transition is indicated with a dashed green line. Upward and downward arrows indicate absorption and emission lines, respectively.

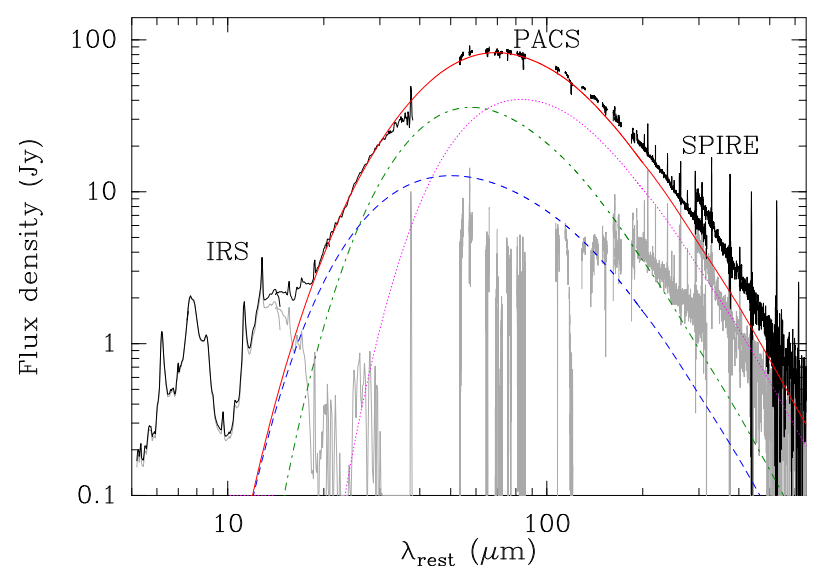

Fig. 3. Spectral energy distribution of Arp 299A from mid-IR to submillimeter wavelengths. Data from PACS and SPIRE on Herschel, and IRS on Spitzer are shown. The models discussed in Sect. 3 are included where the dashed blue and dashed-dotted green curves represent the inner and outer components, respectively. The dotted magenta curve represents the extended component and the solid red curve is the sum of all model components. Gray curves are the residual spectra after model subtraction.

of all spectral ranges observed with PACS and SPIRE are presented in Fig. 3 together with archival data from the Infrared Spectrograph (IRS) on Spitzer (Alonso-Herrero et al. 2009) and the modeled dust continuum discussed in Sect. 3. Spectroscopic parameters for line identification and radiative transfer modeling were obtained from the JPL (Pickett et al. 1998) and CDMS (Müller et al. 2001, 2005) catalogs.

\subsection{Data reduction}

\subsubsection{Observations with PACS}

The data reduction was done with the Herschel interactive processing environment (HIPE; Ott 2010) version 13.0.0 using the standard background normalization pipeline for chopped line scans and short range scans. At a distance of $44.8 \mathrm{Mpc}$, the nuclear far-IR emission in Arp 299A is spatially unresolved in the central 9.4" ( $2 \mathrm{kpc})$ spaxel of the PACS $5 \times 5$ spaxel array. Because the central spaxel is smaller than the point spread function of the spectrometer, the central spectrum was extracted using a point source correction task available in HIPE version 13.0.0 in order to obtain the full flux. In the $\mathrm{OH}^{2} \Pi_{3 / 2}-{ }^{2} \Pi_{3 / 2} \frac{5}{2}-\frac{3}{2}$ doublet at $119 \mu \mathrm{m}$, line absorption and emission are seen outside of the central spaxel, indicating the presence of a spatially more extended, low-excitation molecular component. For this spectral range we have used the sum of the central nine spaxels in order to obtain all the line flux, but this might also introduce contaminating flux from Arp 299B. We finally fitted the continuum and lines in each spectral range with polynomial baselines and Gaussian curves, respectively. Line fluxes, measured and inferred intrinsic Gaussian line widths, and continuum levels for the PACS observations are listed in Table 2. Upper limits on the ${ }^{18} \mathrm{OH}$ and $\mathrm{H}_{2}^{18} \mathrm{O}$ line fluxes were calculated using the instrument resolution assuming a Gaussian profile. The $3 \sigma$ peak flux density limits and flux limits for these lines are presented in Table 3.

\subsubsection{Observations with SPIRE}

The data reduction was done with the standard single pointing pipeline in HIPE 13.0.0. There is a noticeable mismatch in the continuum level in the overlap region between the two SPIRE detectors (see Fig. 3). This is due to a discontinuity in the wavelength dependent beam size between the detectors, and indicates that there is flux outside of the beam of the short wavelength array. This might mean that the source is actually partially extended, or that the detector with the larger beam size picks up flux from the other components of the system (see Fig. 1). We expect that the $\mathrm{H}_{2} \mathrm{O}$ emission lines that are of interest here arise in a relatively compact region around the nucleus of Arp 299A (though more extended than the absorption lines, see Sect. 3), but line emission from Arp 299B/C is also possible. The flux in the $\mathrm{H}_{2} \mathrm{O} 303 \mu \mathrm{m} 2_{02} \rightarrow 1_{11}$ line, which is located in the overlap region of SPIRE, shows $\sim 30 \%$ more flux in the long wavelength array than in the short wavelength array. To avoid contamination from Arp 299B/C we have used the flux from the short wavelength array, which has the smaller beam size. The full-widthhalf-maximum of the beam at the wavelength of the $\mathrm{H}_{2} \mathrm{O} 399 \mu \mathrm{m}$ $2_{11} \rightarrow 2_{02}$ line, which is only detected by the long wavelength array, is $\sim 40^{\prime \prime}$, and it is likely contaminated by flux from the other components of the system.

We extracted line fluxes using FTFitter $^{1}$, an IDL based line fitter for Fourier transform spectrometer (FTS) spectra. A polynomial baseline was subtracted from the spectrum of each detector and the spectral lines were then simultaneously fitted using Gaussian profiles convolved with the FTS instrumental line shape (a sinc function). Unresolved lines were fitted using the instrumental line shape only. Line fluxes and continuum levels for the $\mathrm{H}_{2} \mathrm{O}$ lines observed with SPIRE are listed in Table 4. Upper limits on the $\mathrm{H}_{2}^{18} \mathrm{O}$ line fluxes in the SPIRE range were calculated using a sinc function assuming the instrumental resolution. The $3 \sigma$ peak flux density limits and flux limits for those $\mathrm{H}_{2}^{18} \mathrm{O}$ transitions that are well separated from other strong lines are presented in Table 3.

\section{2. $\mathrm{H}_{2} \mathrm{O}$}

Nine $\mathrm{H}_{2} \mathrm{O}$ absorption lines and one emission line were detected with PACS. The single emission line lies at $187 \mu \mathrm{m}$, the longest wavelength of all $\mathrm{H}_{2} \mathrm{O}$ lines detected with PACS. The absorptions have lower (pre-transition) energy levels up to $\sim 650 \mathrm{~K}$ while the emission line has an upper energy level of $\sim 400 \mathrm{~K}$.

\footnotetext{
1 https://www $\cdot$ uleth.ca/phy/naylor/index $\cdot$ php?page= ftfitter
} 
Table 1. Summary of Herschel observations.

\begin{tabular}{lcccc}
\hline \hline Instrument & Observation ID & $\begin{array}{c}\text { Date } \\
\text { YYY-MM-DD }\end{array}$ & $\begin{array}{c}\text { Duration } \\
(\mathrm{s})\end{array}$ & $\begin{array}{c}\text { Wavelength ranges (rest) } \\
(\mu \mathrm{m})\end{array}$ \\
\hline PACS & 1342232605 & $2011-11-21$ & 976 & $117.6-120.7$ \\
PACS & 1342232607 & $2011-11-21$ & 4759 & $184.3-188.6$ \\
PACS & 1342254239 & $2012-10-31$ & 841 & $53.4-54.8,68.7-70.0$ \\
& & & & $106.7-109.5,137.3-139.9$ \\
PACS & 1342254240 & $2012-10-31$ & 3028 & $80.9-82.5,82.3-84.2,83.8-85.5$ \\
& & & & $161.7-165.0,164.7-168.4,167.6-171.0$ \\
PACS & 1342254241 & $2012-10-31$ & 1151 & $57.1-58.4,64.5-66.1$ \\
& & & & $114.3-116.8,128.9-132.1$ \\
PACS & 1342254242 & $2012-10-31$ & 2198 & $70.4-72.4,74.8-75.9,75.8-77.2$ \\
& & & & $140.8-144.9,149.5-151.7,151.5-154.4$ \\
SPIRE & 1342199248 & $2010-06-27$ & 4964 & $192-310,300-664$ \\
\hline
\end{tabular}

Table 2. $\mathrm{H}_{2} \mathrm{O}$ and $\mathrm{OH}$ lines detected with PACS.

\begin{tabular}{|c|c|c|c|c|c|c|c|c|}
\hline Line & $\begin{array}{c}E_{\text {lower }} \\
(\mathrm{K})\end{array}$ & $\begin{array}{l}\lambda_{\text {rest }} \\
(\mu \mathrm{m})\end{array}$ & $\begin{array}{c}V_{\mathrm{c}}^{a, b} \\
\left(\mathrm{~km} \mathrm{~s}^{-1}\right)\end{array}$ & $\begin{array}{c}\Delta V^{a, c} \\
\left(\mathrm{~km} \mathrm{~s}^{-1}\right)\end{array}$ & $\begin{array}{c}\Delta V_{\text {inf }^{d}} \\
\left(\mathrm{~km} \mathrm{~s}^{-1}\right)\end{array}$ & $\begin{array}{l}\text { Cont. }^{e} \\
\text { (Jy) }\end{array}$ & $\begin{array}{c}\text { Line peak } \\
(\mathrm{Jy})\end{array}$ & $\begin{array}{c}\text { Flux }^{a, f} \\
\left(\mathrm{Jy} \mathrm{km} \mathrm{s}^{-1}\right)\end{array}$ \\
\hline $\mathrm{H}_{2} \mathrm{O} 4_{22} \rightarrow 3_{13}$ & 205 & 57.636 & $-7(12)$ & $346(30)$ & 280 & 83.2 & $-3.0(0.2)$ & $-1071(117)$ \\
\hline $\mathrm{H}_{2} \mathrm{O} 5_{24} \rightarrow 4_{13}$ & 396 & 71.067 & $34(25)$ & $283(62)$ & 229 & 81.2 & $-1.6(0.3)$ & $-476(133)$ \\
\hline $\mathrm{H}_{2} \mathrm{O} 7_{17} \rightarrow 6_{06}$ & 643 & 71.540 & $-4(24)$ & $147(59)$ & $\ldots$ & 82.3 & $-0.9(0.3)$ & $-148(78)$ \\
\hline $\mathrm{H}_{2} \mathrm{O} 7_{07} \rightarrow 6_{16}$ & 644 & 71.947 & $-42(22)$ & $281(56)$ & 228 & 83.0 & $-1.3(0.2)$ & $-396(103)$ \\
\hline $\mathrm{H}_{2} \mathrm{O} 3_{21} \rightarrow 2_{12}$ & 114 & 75.381 & $36(2)$ & $320(6)$ & 279 & 85.0 & $-11.9(0.2)$ & $-4032(96)$ \\
\hline $\mathrm{H}_{2} \mathrm{O} 6_{16} \rightarrow 5_{05}$ & 468 & 82.031 & $15(16)$ & $358(39)$ & 330 & 80.2 & $-2.4(0.2)$ & $-900(126)$ \\
\hline $\mathrm{H}_{2} \mathrm{O} 6_{06} \rightarrow 5_{15}$ & 470 & 83.284 & $-8(17)$ & $272(42)$ & 235 & 79.7 & $-2.0(0.3)$ & $-578(115)$ \\
\hline $\mathrm{H}_{2} \mathrm{O} 2_{21} \rightarrow 1_{10}$ & 61 & 108.073 & $15(7)$ & $386(17)$ & 226 & 65.5 & $-5.7(0.2)$ & $-2348(133)$ \\
\hline $\mathrm{H}_{2} \mathrm{O}_{13} \rightarrow 2_{02}$ & 101 & 138.528 & $-33(21)$ & $446(53)$ & 356 & 42.6 & $-1.3(0.1)$ & $-600(93)$ \\
\hline $\mathrm{H}_{2} \mathrm{O} 4_{13} \rightarrow 4_{04}$ & 320 & 187.111 & $-53(22)$ & $385(55)$ & 343 & 23.0 & $1.1(0.2)$ & $469(87)$ \\
\hline $\mathrm{OH}^{2} \Pi_{3 / 2}-{ }^{2} \Pi_{3 / 2} \frac{9}{2}^{-}-\frac{7}{2}^{+}$ & 291 & 65.132 & $13(5)$ & $387(13)$ & 341 & 86.6 & $-8.3(0.2)$ & $-3432(145)$ \\
\hline $\mathrm{OH}^{2} \Pi_{3 / 2}-{ }^{2} \Pi_{3 / 2} \frac{9}{2}^{+}-\frac{7}{2}^{-}$ & 291 & 65.279 & $8(5)$ & $362(12)$ & 313 & 86.6 & $-8.6(0.2)$ & $-3331(138)$ \\
\hline $\mathrm{OH}^{2} \Pi_{1 / 2}-{ }^{2} \Pi_{1 / 2} \frac{7}{2}-\frac{5}{2}^{2}$ & 416 & $71.197^{g}$ & $31(8)$ & $405(22)$ & $\ldots$ & 81.4 & $-5.4(0.2)$ & $-2311(159)$ \\
\hline $\mathrm{OH}^{2} \Pi_{3 / 2}-^{2} \Pi_{3 / 2} \frac{7}{2}^{+}-\frac{5}{2}^{-}$ & 121 & 84.420 & $23(3)$ & $321(8)$ & 292 & 79.5 & $-14.9(0.3)$ & $-5085(159)$ \\
\hline $\mathrm{OH}^{2} \Pi_{3 / 2}-{ }^{2} \Pi_{3 / 2} \frac{7}{2}^{-}-\frac{5}{2}^{+}$ & 121 & 84.597 & $39(3)$ & $309(8)$ & 278 & 79.5 & $-15.4(0.3)$ & $-5073(160)$ \\
\hline $\mathrm{OH}^{2} \Pi_{3 / 2}-{ }^{2} \Pi_{3 / 2} \frac{5}{2}^{-}-\frac{3}{2}^{+}$ & 0 & 119.233 & $172(8)$ & $241(18)$ & $\ldots$ & 73.9 & $-7.3(0.5)$ & $-1871(187)$ \\
\hline $\mathrm{OH}^{2} \Pi_{3 / 2}-^{2} \Pi_{3 / 2} \frac{5}{2}^{+}-\frac{3}{2}^{-}$ & 0 & 119.441 & $172(8)$ & $238(20)$ & $\ldots$ & 73.9 & $-6.8(0.5)$ & $-1722(187)$ \\
\hline $\mathrm{OH}^{2} \Pi_{1 / 2}-{ }^{2} \Pi_{1 / 2} \frac{3}{2}^{+}-\frac{1}{2}^{-}$ & 182 & 163.126 & $11(11)$ & $413(27)$ & 343 & 31.4 & $4.9(0.2)$ & $2148(157)$ \\
\hline $\mathrm{OH}^{2} \Pi_{1 / 2}-^{2} \Pi_{1 / 2} \frac{3}{2}^{-}-\frac{1}{2}^{+}$ & 182 & 163.397 & $33(10)$ & $417(23)$ & 347 & 31.4 & $5.3(0.2)$ & $2373(153)$ \\
\hline
\end{tabular}

Notes. ${ }^{(a)}$ Values from Gaussian fits to the lines. Numbers in parenthesis indicate $1 \sigma$ uncertainties from these fits. ${ }^{(b)}$ Velocity shift of line center relative to $z=0.010411$. $^{(c)}$ FWHM of lines. ${ }^{(d)}$ Inferred velocity width is based on the instrument resolution assuming a Gaussian profile; this is not listed for doublets that are not well separated. ${ }^{(e)}$ Value of the fitted baseline at the line center. ${ }^{(f)}$ Absorption lines indicated with minus sign. (g) The two $\Lambda$-components are (almost) blended into a single spectral feature.

The spectral ranges around the lines are shown in Fig. 4, the spectral line energy distribution is presented in Fig. 5, and the measured line parameters are summarized in Table 2. There is no systematic velocity shift among the $\mathrm{H}_{2} \mathrm{O}$ lines and within $2 \sigma$ all but one of the lines are consistent with the galaxy redshift of $z=0.010411$ (Nordgren et al. 1997). The $\mathrm{H}_{2} \mathrm{O} 139 \mu \mathrm{m}$ $3_{13} \rightarrow 2_{02}$ line has a possible blueshifted line wing extending down to $\sim-600 \mathrm{~km} \mathrm{~s}^{-1}$, but this spectral range is noisy and the feature is at the $1 \sigma$-level. We see no obvious contamination by other species in any of the lines.

Seven $\mathrm{H}_{2} \mathrm{O}$ transitions, with upper energy levels up to $\sim 650 \mathrm{~K}$, were detected in emission with SPIRE. The line fluxes are summarized in Table 4, the spectral ranges around the lines are presented in Fig. 6, and the spectral line energy distribution is shown in Fig. 7. One of the transitions, $\mathrm{H}_{2} \mathrm{O} 399 \mu \mathrm{m} 2_{11} \rightarrow 2_{02}$, was detected with the spectrometer long wavelength array; due to the larger beam size for this detector, this line might be contaminated by flux from other parts of Arp 299. No $\mathrm{H}_{2}^{18} \mathrm{O}$ lines were detected (see Table 3.)

\section{3. $\mathrm{OH}$}

Five $\mathrm{OH}$ doublets, with lower energy levels up to $\sim 400 \mathrm{~K}$, were detected with PACS. The doublets were mainly seen in absorption, but the $\mathrm{OH}{ }^{2} \Pi_{1 / 2}-{ }^{2} \Pi_{1 / 2} \frac{3}{2}-\frac{1}{2}$ doublet at $163 \mu \mathrm{m}$ was detected in emission. This doublet is often seen in emission, 


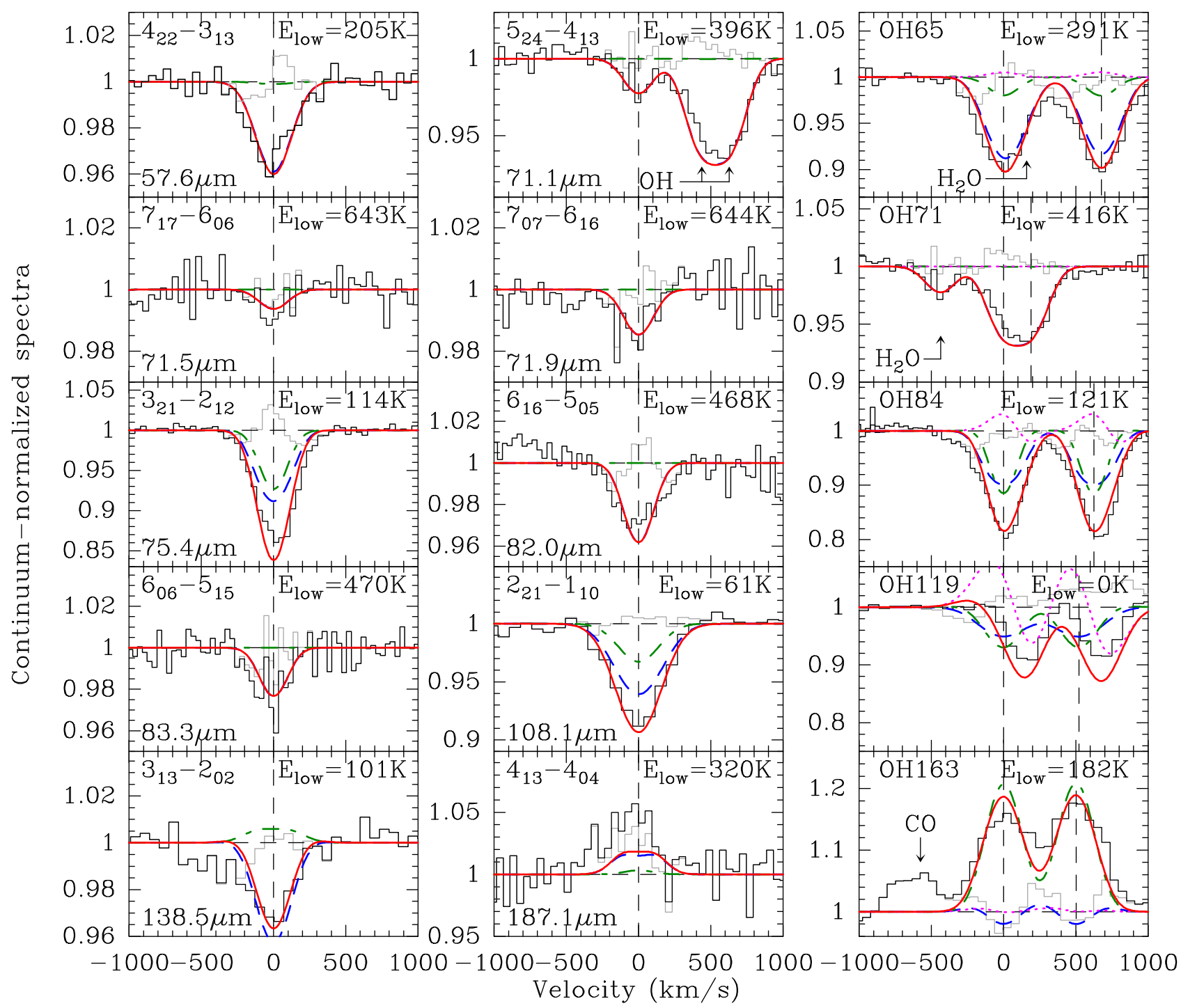

Fig. 4. $\mathrm{H}_{2} \mathrm{O}$ and $\mathrm{OH}$ lines observed in Arp 299A with PACS. The black histograms are the observed, continuum-normalized, spectra. The velocity scale for the $\mathrm{OH}$ lines refers to the blue component in each doublet. The best fit models from Sect. 3 are also included. The dashed blue and dashed-dotted green curves denote the models for the inner and outer components, respectively. The dotted magenta curve in the OH spectra represents the tentative extended component and the solid red curve denotes the sum of the models. Gray histograms are the residual spectra after model subtraction.

for example in NGC 4418 and Arp 220 (G-A12), because it is usually excited by absorption in the $\mathrm{OH}^{2} \Pi_{1 / 2}-{ }^{2} \Pi_{3 / 2} \frac{5}{2}-\frac{3}{2}$ and ${ }^{2} \Pi_{1 / 2}-{ }^{2} \Pi_{3 / 2} \frac{3}{2}-\frac{3}{2}$ doublets at 35 and $53.3 \mu \mathrm{m}$, respectively, followed by a cascade down to the ground state. Measured line parameters of the detected doublets are summarized in Table 2 and the spectral ranges containing the lines are shown in Fig. 4. All lines are also indicated in the energy diagram of $\mathrm{OH}$ in Fig. 2. Most lines seem to be uncontaminated by other species, but the $\mathrm{OH}^{2} \Pi_{3 / 2}-{ }^{2} \Pi_{3 / 2} \frac{9}{2}^{-}-\frac{7}{2}^{+}$line at $65 \mu \mathrm{m}$ might be weakly contaminated by $\mathrm{H}_{2} \mathrm{O}^{2} 6_{25} \stackrel{2}{\rightarrow} 514$. The extended line emission and absorption seen in the $\mathrm{OH}^{2} \Pi_{3 / 2}-{ }^{2} \Pi_{3 / 2} \frac{5}{2}-\frac{3}{2}$ doublet at $119 \mu \mathrm{m}$ combined with the strong redshift $\left(\sim 175 \mathrm{~km} \mathrm{~s}^{-1}\right)$ in its absorption indicates the presence of an extended component with inflowing molecular gas. No ${ }^{18} \mathrm{OH}$ doublets were detected (see Table 3). We do not estimate an upper limit of the blue component of the ${ }^{18} \mathrm{OH}^{2} \Pi_{3 / 2}-{ }^{2} \Pi_{3 / 2} \frac{5}{2}-\frac{3}{2}$ doublet at $120 \mu \mathrm{m}$ as it is close to an absorption in the $\mathrm{CH}^{+} 3-2$ line. It is possible that some of the flux in this absorption comes from the blue component of the ${ }^{18} \mathrm{OH}$ doublet, but we find this unlikely as the red component of this doublet is missing while the components of the ${ }^{16} \mathrm{OH}^{2} \Pi_{3 / 2}-{ }^{2} \Pi_{3 / 2} \frac{5}{2}-\frac{3}{2}$ doublet at $119 \mu \mathrm{m}$ show almost equal strengths.

\section{Models}

We have modeled the observations using the spherically symmetric radiative transfer code described by González-Alfonso \& Cernicharo (1997, 1999), including a treatment of line overlaps for the $\mathrm{OH} \Lambda$-components. Dust emission is included with the dust grains being simulated as a mixture of silicates and amorphous carbon, for which the adopted mass absorption coefficient as a function of wavelength is shown in González-Alfonso et al. (2014a). The model parameters used to characterize the physical conditions in the source are the dust opacity at $100 \mu \mathrm{m}\left(\tau_{100}\right)$, the dust temperature $\left(T_{\text {dust }}\right)$, the gas temperature $\left(T_{\text {gas }}\right)$, the $\mathrm{H}_{2}$ density $\left(n_{\mathrm{H}_{2}}\right)$, and the column density of $\mathrm{H}_{2} \mathrm{O}$ or $\mathrm{OH}$ per optical depth at $50 \mu \mathrm{m}\left(N_{\mathrm{H}_{2} \mathrm{O} / \mathrm{OH}} / \tau_{50}\right)$. Collisional rates with $\mathrm{H}_{2}$ are taken from Dubernet et al. (2009) and Daniel et al. (2011) for $\mathrm{H}_{2} \mathrm{O}$ and from Offer et al. (1994) for OH. Line broadening is simulated 
Table 3. $3 \sigma$ upper limits to undetected $\mathrm{H}_{2}^{18} \mathrm{O}$ and ${ }^{18} \mathrm{OH}$ lines.

\begin{tabular}{|c|c|c|c|c|}
\hline Line & $\begin{array}{l}\lambda_{\text {rest }} \\
(\mu \mathrm{m})\end{array}$ & $\begin{array}{c}\text { Line peak }^{a} \\
\quad(\mathrm{Jy})\end{array}$ & $\begin{array}{c}\text { Flux }^{a, b} \\
\left(\mathrm{Jy} \mathrm{km} \mathrm{s}^{-1}\right)\end{array}$ & $\begin{array}{l}\text { Predicted flux } \\
\left(\mathrm{Jy} \mathrm{km} \mathrm{s}^{-1}\right)\end{array}$ \\
\hline $\mathrm{H}_{2}^{18} \mathrm{O} 4_{22} \rightarrow 3_{13}$ & 57.840 & $<0.99$ & $<210$ & $\ldots$ \\
\hline $\mathrm{H}_{2}^{18} \mathrm{O} 5_{24} \rightarrow 4_{13}$ & 71.754 & $<1.20$ & $<210$ & $\ldots$ \\
\hline $\mathrm{H}_{2}^{18} \mathrm{O} 6_{06} \rightarrow 5_{15}$ & 83.591 & $<1.19$ & $<170$ & $\ldots$ \\
\hline $\mathrm{H}_{2}^{18} \mathrm{O} 3_{13} \rightarrow 2_{02}$ & 139.586 & $<1.32$ & $<370$ & $\ldots$ \\
\hline $\mathrm{H}_{2}^{18} \mathrm{O} 2_{20} \rightarrow 2_{11}$ & 250.034 & $<0.54$ & $<160$ & $\ldots$ \\
\hline $\mathrm{H}_{2}^{18} \mathrm{O} 4_{22} \rightarrow 4_{13}$ & 252.167 & $<0.51$ & $<150$ & $\ldots$ \\
\hline $\mathrm{H}_{2}^{18} \mathrm{O} 3_{12} \rightarrow 3_{03}$ & 253.762 & $<0.48$ & $<140$ & $\ldots$ \\
\hline $\mathrm{H}_{2}^{18} \mathrm{O} 3_{21} \rightarrow 3_{12}$ & 263.738 & $<0.51$ & $<160$ & $\ldots$ \\
\hline${ }^{18} \mathrm{OH}^{2} \Pi_{3 / 2}-{ }^{2} \Pi_{3 / 2} \frac{9}{2}^{-}-\frac{7}{2}^{+}$ & 65.543 & $<1.59$ & $<310$ & -40 \\
\hline${ }^{18} \mathrm{OH}^{2} \Pi_{3 / 2}-{ }^{2} \Pi_{3 / 2} \frac{9}{2}^{+}-\frac{7}{2}^{-}$ & 65.690 & $<1.59$ & $<310$ & -40 \\
\hline${ }^{18} \mathrm{OH}^{2} \Pi_{1 / 2}-{ }^{2} \Pi_{1 / 2} \frac{7}{2}-\frac{5}{2}{ }^{2}$ & 71.679 & $<1.05$ & $<190$ & -10 \\
\hline${ }^{18} \mathrm{OH}^{2} \Pi_{3 / 2}-{ }^{2} \Pi_{3 / 2} \frac{7}{2}^{+}-\frac{5}{2}^{-}$ & 84.947 & $<1.62$ & $<230$ & -230 \\
\hline${ }^{18} \mathrm{OH}^{2} \Pi_{3 / 2}-{ }^{2} \Pi_{3 / 2} \frac{\overline{7}}{2}^{-}-\frac{5}{2}^{+}$ & 85.123 & $<1.62$ & $<230$ & -230 \\
\hline${ }^{18} \mathrm{OH}^{2} \Pi_{3 / 2}-^{2} \Pi_{3 / 2} \frac{5}{2}^{+}-\frac{3}{2}^{-}$ & $120.171^{d}$ & $<1.23$ & $<380$ & -330 \\
\hline${ }^{18} \mathrm{OH}^{2} \Pi_{1 / 2}-{ }^{2} \Pi_{1 / 2} \frac{3}{2}^{+}-\frac{1}{2}^{-}$ & 164.269 & $<0.90$ & $<220$ & 30 \\
\hline${ }^{18} \mathrm{OH}^{2} \Pi_{1 / 2}-{ }^{2} \Pi_{1 / 2} \frac{3}{2}^{-}-\frac{1}{2}^{+}$ & 164.545 & $<0.90$ & $<220$ & 30 \\
\hline
\end{tabular}

Notes. ${ }^{(a)}$ Upper limits are to the absolute values. ${ }^{(b)}$ Upper limits to the fluxes were calculated using the instrument resolution and assuming a Gaussian profile for lines observed with PACS and a sinc profile for lines observed with SPIRE. ${ }^{(c)}$ Predicted flux, for the ${ }^{18} \mathrm{OH}$ lines, from the inner component using the limiting value $N_{\mathrm{OH}} / \tau_{50}=5 \times 10^{15}$, corresponding to a lower limit on the ${ }^{16} \mathrm{O} /{ }^{18} \mathrm{O}$ ratio of 400 . ${ }^{(d)}$ The blue component of the ${ }^{18} \mathrm{OH}$ doublet at $120 \mu \mathrm{m}$ is close to the $\mathrm{CH}^{+} 3-2$ absorption line and we only estimate an upper limit for the red component.

Table 4. $\mathrm{H}_{2} \mathrm{O}$ lines detected with SPIRE.

\begin{tabular}{lcccccccc}
\hline \hline Line & $\begin{array}{c}E_{\text {upper }} \\
(\mathrm{K})\end{array}$ & $\begin{array}{c}\lambda_{\text {rest }} \\
(\mu \mathrm{m})\end{array}$ & $\begin{array}{c}V_{\mathrm{c}}^{a, b} \\
\left(\mathrm{~km} \mathrm{~s}^{-1}\right)\end{array}$ & $\begin{array}{c}\Delta V^{a, c} \\
\left(\mathrm{~km} \mathrm{~s}^{-1}\right)\end{array}$ & $\begin{array}{c}\Delta V_{\text {inf }^{d}} \\
\left(\mathrm{~km} \mathrm{~s}^{-1}\right)\end{array}$ & $\begin{array}{c}\text { Cont. }^{e} \\
(\mathrm{Jy})\end{array}$ & $\begin{array}{c}\text { Line peak }^{a} \\
(\mathrm{Jy})\end{array}$ & $\begin{array}{c}\text { Flux }^{a} \\
\left(\mathrm{Jy} \mathrm{km} \mathrm{s}^{-1}\right)\end{array}$ \\
\hline $\mathrm{H}_{2} \mathrm{O} 1_{11} \rightarrow 0_{00}{ }^{f}$ & 53 & 269.272 & $\ldots$ & $\ldots$ & $\ldots$ & 8.1 & $<0.18$ & $<57$ \\
$\mathrm{H}_{2} \mathrm{O} \mathrm{2} 2_{02} \rightarrow 1_{11}$ & 101 & 303.456 & $-24(12)$ & $382(36)$ & $\ldots$ & 5.7 & $2.6(0.2)$ & $891(53)$ \\
$\mathrm{H}_{2} \mathrm{O} 2_{11} \rightarrow 2_{02}{ }^{g}$ & 137 & 398.643 & $-4(26)$ & $516(90)$ & $\ldots$ & 1.7 & $1.7(0.3)$ & $809(80)$ \\
$\mathrm{H}_{2} \mathrm{O} 2_{20} \rightarrow 2_{11}$ & 196 & 243.974 & $12(18)$ & $367(47)$ & $309(59)$ & 10.9 & $1.8(0.2)$ & $652(156)$ \\
$\mathrm{H}_{2} \mathrm{O} \mathrm{3} 3_{12} \rightarrow 3_{03}$ & 249 & 273.193 & $-25(15)$ & $457(41)$ & $415(44)$ & 7.8 & $2.4(0.2)$ & $1132(153)$ \\
$\mathrm{H}_{2} \mathrm{O} \mathrm{3} 3_{21} \rightarrow 3_{12}$ & 305 & 257.795 & $-11(8)$ & $375(21)$ & $304(28)$ & 9.3 & $4.1(0.2)$ & $1528(178)$ \\
$\mathrm{H}_{2} \mathrm{O} 4_{22} \rightarrow 413$ & 454 & 248.247 & $-4(20)$ & $389(55)$ & $322(65)$ & 10.4 & $1.6(0.2)$ & $615(155)$ \\
$\mathrm{H}_{2} \mathrm{O} 5_{23} \rightarrow 5_{14}$ & 642 & 212.526 & $-22(34)$ & $550(88)$ & $546(82)$ & 16.3 & $1.1(0.2)$ & $628(125)$ \\
\hline
\end{tabular}

Notes. ${ }^{(a)}$ Values from fits to the spectral lines, numbers in parenthesis indicate $1 \sigma$ uncertainties from these fits. ${ }^{(b)}$ Velocity shift of line center relative to $z=0.010411 .^{(c)}$ FWHM of lines. ${ }^{(d)}$ Inferred velocity width is based on the instrument resolution assuming a sinc profile, only listed for partially resolved lines. ${ }^{(e)}$ Value of the fitted baseline at the line center. ${ }^{(f)}$ Undetected, upper limit is $1 \sigma$ value. ${ }^{(g)}$ Detected with the spectrometer long wavelength array, due to the larger beam size this line might be contaminated by flux from the other components of Arp 299.

by a microturbulent velocity $\left(v_{\text {tur }}\right)$ which is set to $80 \mathrm{~km} \mathrm{~s}^{-1}$ in order to match the observed line widths, regardless of whether it is due to turbulence or velocity gradients along the line of sight. Our results on line ratios depend on $N_{\mathrm{H}_{2} \mathrm{O} / \mathrm{OH}} / \Delta V$. If rotation is (partially) responsible for line broadening, the velocity dispersion along each line of sight would be somewhat lower and thus $\mathrm{N}_{\mathrm{H}_{2} \mathrm{O} / \mathrm{OH}}$ would be somewhat decreased. The column density ratios would be the same, but abundances would be somewhat lower. In all models we have adopted a gas-to-dust ratio of 100 by mass, similar to the average value in LIRGs reported by Wilson et al. (2008). We have assumed a covering factor of unity for all models.

The data presented in Sect. 2 reveal a rich molecular line spectrum where the $\mathrm{OH}$ and $\mathrm{H}_{2} \mathrm{O}$ lines in the far-IR PACS spectra are seen primarily in absorption while the $\mathrm{H}_{2} \mathrm{O}$ lines in the submm SPIRE spectrum are seen in emission only. This division of absorption and emission lines between PACS and SPIRE is caused by the excitation of $\mathrm{H}_{2} \mathrm{O}$ rotational levels through absorption in the far-IR lines, followed by de-excitation through emission in lines at longer (submm) wavelengths where the optical depth of the dust is lower. Similar absorption/emission dichotomies are seen in Mrk 231 (González-Alfonso et al. 2010) and $\mathrm{Zw} 049.057$ (Falstad et al. 2015) and the excitation processes for the $\mathrm{H}_{2} \mathrm{O}$ submm lines are further explored in González-Alfonso et al. (2014a). The rich variety of emission and absorption lines cannot be reproduced by a single set of parameters as the high- and low-lying lines are expected to arise in different regions due to their differing excitation requirements. Similar to the inner regions of Arp 220, NGC 4418, and Zw 049.057 (G-A12; Falstad et al. 2015) the high excitation 


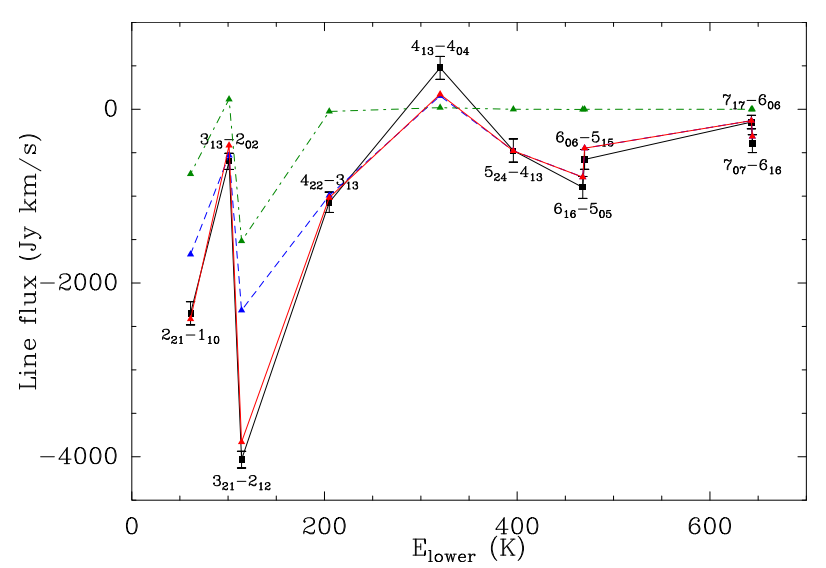

Fig. 5. Spectral line energy distribution of the $\mathrm{H}_{2} \mathrm{O}$ lines detected with PACS, the black squares represent the data. Model predictions are included with the dashed blue and dashed-dotted green curves denoting the models for the inner and outer components, respectively. The solid red curve is the sum of these two models.

seen in both $\mathrm{H}_{2} \mathrm{O}$ and $\mathrm{OH}$ in Arp 299A cannot be accounted for by collisions alone. The dominant excitation mechanism is instead absorption of photons emitted by the warm dust in the central regions of the galaxy. In our modeling we try to reproduce the observed molecular absorption/emission using the least possible number of parameterized components and find that the lines are primarily formed in two components, which are discussed in Sects. 3.2 and 3.3 and summarized in Tables 5 and 6. A tentative additional component which can account for the redshifted ground state $\mathrm{OH}^{2} \Pi_{3 / 2}-{ }^{2} \Pi_{3 / 2} \frac{5}{2}-\frac{3}{2}$ doublet at $119 \mu \mathrm{m}$ is discussed in Sect. 3.4. All three components are included in a schematic representation of the model in Fig. 8. A short outline of the modeling is presented in Sect. 3.1.

\subsection{Outline of the modeling}

Our general approach to the modeling was to compare the observed ratios of various $\mathrm{H}_{2} \mathrm{O}$ lines to a grid of models and then estimate the radius $(R)$ from the scaling to the total flux of the lines. High-lying $\left(E_{\text {lower }} \gtrsim 300 \mathrm{~K}\right)$ lines were fitted first as they are expected to be uncontaminated by absorption from a lowerexcitation component. To account for the missing flux in the lowlying lines, the procedure was then repeated for them, but now taking the contribution from the first component into account. Finally, the $\mathrm{OH}$ lines were reproduced by fitting an $\mathrm{OH}$ column to the models developed for $\mathrm{H}_{2} \mathrm{O}$. The details of the modeling in the two components are discussed in Sects. 3.2 and 3.3. An approach where the inner and outer model components were fitted simultaneously was also tested, and yielded similar results.

\subsection{Inner component}

The high-lying $\left(E_{\text {lower }} \gtrsim 300 \mathrm{~K}\right.$ ) lines of $\mathrm{H}_{2} \mathrm{O}$ provide the best constraints for the parameters in the inner regions of the galaxy. We have compared the observed fluxes of the five highest-lying lines detected with PACS and the two highest-lying lines detected with SPIRE (see Figs. 4-7), all normalized to the flux of the $\mathrm{H}_{2} \mathrm{O} 58 \mu \mathrm{m} 4_{22} \rightarrow 3_{13}$ line, to a grid of models where we have varied the parameters $\tau_{100}, T_{\text {dust }}, T_{\text {gas }}, n_{\mathrm{H}_{2}}$, and $N_{\mathrm{H}_{2} \mathrm{O}} / \tau_{50}$. In each grid point we have computed the reduced $\chi^{2}$ for the line ratios:

$\chi^{2}=\frac{1}{N-n} \sum_{i=1}^{N} \frac{\left(\mathrm{obs}_{i}-\operatorname{model}_{i}\right)^{2}}{\sigma_{i}^{2}}$, were $N$ is the number of line ratios fitted, $n$ is the number of fitted parameters, obs ${ }_{i}$ and model $_{i}$ are the observed and modeled values of line ratio $i$, respectively, and $\sigma_{i}$ is the standard deviation of line ratio $i$, calculated through error propagation. Parameter ranges are determined based on where the $\chi^{2}$ has increased by at least $20 \%$ from its minimum value.

We find that the best fit to the observations is achieved with a dust opacity of $\tau_{100}=1-4$, with $\tau_{100}=2$ being the preferred value. Because of the high values of the Einstein coefficients of the $\mathrm{OH}$ and $\mathrm{H}_{2} \mathrm{O}$ lines, for values of $n_{\mathrm{H}_{2}} \leq 3 \times 10^{6} \mathrm{~cm}^{-3}$ collisions have negligible impact on the results. In Fig. 9 we show the reduced $\chi^{2}$ in our grid of models for the preferred dust opacity of $\tau_{100}=2$. The relative fluxes of the high-lying lines can be well fitted with different combinations of dust temperatures between $T_{\text {dust }}=90$ and $120 \mathrm{~K}$, and $\mathrm{H}_{2} \mathrm{O}$ columns between $N_{\mathrm{H}_{2} \mathrm{O}} / \tau_{50}=5 \times 10^{17}$ and $2 \times 10^{18} \mathrm{~cm}^{-2}$, with lower $T_{\text {dust }}$ corresponding to higher $N_{\mathrm{H}_{2} \mathrm{O}} / \tau_{50}$. To account for the absolute flux in the lines, a radius of the component between $R=20$ and $25 \mathrm{pc}$ is required. All parameter ranges are summarized in Tables 5 and 6 . The best fit, which is also included in Figs. 3-7, is achieved with $\tau_{100}=2, T_{\text {dust }}=100 \mathrm{~K}, N_{\mathrm{H}_{2} \mathrm{O}} / \tau_{50}=1 \times 10^{18} \mathrm{~cm}^{-2}$ and $R=22 \mathrm{pc}$. This component accounts for both the high-lying absorption lines in the far-IR and the high-lying $\left(E_{\text {upper }}>400 \mathrm{~K}\right)$ emission lines in the submillimeter. The column density of $\mathrm{H}_{2}$ is calculated from the value of $\tau_{100}$ assuming a mass-absorption coefficient of $44.4 \mathrm{~cm}^{2} \mathrm{~g}^{-1}$ at $100 \mu \mathrm{m}$ and a gas-to-dust mass ratio of 100. The molecular mass of the component is then calculated from this value, assuming spherical symmetry. Finally, the dust luminosity is based on the radius, temperature, and opacity of the component.

$\mathrm{OH}^{2} \Pi_{1 / 2}-{ }^{2} \Pi_{1 / 2} \frac{7}{2}-\frac{5}{2}$ at $71 \mu \mathrm{m}$, the transition with the highest lower-state energy, is the only doublet that is expected to be formed in the inner component only. The $\chi^{2}$ for the $\mathrm{OH} 71 \mu \mathrm{m}$ ${ }^{2} \Pi_{1 / 2}-{ }^{2} \Pi_{1 / 2} \frac{7}{2}-\frac{5}{2}$ is presented in Fig. 10 for different values of $N_{\mathrm{OH}} / \tau_{50}$ applied to the best fit model found for $\mathrm{H}_{2} \mathrm{O}$. We find that the flux is best reproduced in the range $N_{\mathrm{OH}} / \tau_{50}=2 \times 10^{18}$ to $1 \times 10^{19} \mathrm{~cm}^{-2}$, corresponding to an $\mathrm{OH} / \mathrm{H}_{2} \mathrm{O}$ ratio in the range 2 to 10 .

An upper limit to the ${ }^{18} \mathrm{OH}$ column density was estimated using the $3 \sigma$ flux upper limits of the three lowest-lying doublets ${ }^{18} \mathrm{OH}^{2} \Pi_{3 / 2}-{ }^{2} \Pi_{3 / 2} \frac{7}{2}-\frac{5}{2},{ }^{2} \Pi_{3 / 2}-{ }^{2} \Pi_{3 / 2} \frac{5}{2}-\frac{3}{2}$, and ${ }^{2} \Pi_{1 / 2}-{ }^{2} \Pi_{1 / 2} \frac{3}{2}-$ $\frac{1}{2}$ at 85,120 , and $164 \mu \mathrm{m}$, respectively. The non-detections of these doublets are not compatible with an ${ }^{18} \mathrm{OH}$ column density higher than $N_{18} \mathrm{OH} / \tau_{50}=5 \times 10^{15} \mathrm{~cm}^{-2}$, corresponding to a lower limit of 400 to the ${ }^{16} \mathrm{OH} /{ }^{18} \mathrm{OH}$ ratio. Predicted flux values using this limiting value are included in Table 3 .

\subsection{Outer component}

Even in the best fit model for the inner component there is flux missing in the low-lying $\mathrm{OH}$ and and $\mathrm{H}_{2} \mathrm{O}$ lines detected with PACS and, especially, the low-lying $\mathrm{H}_{2} \mathrm{O}$ lines detected with SPIRE. To account for this missing flux in the models, a less excited and more extended component is required. To constrain the parameters of this outer component we have followed the same general procedure as for the inner component, but now for the low-lying lines normalized to the flux of the $\mathrm{H}_{2} \mathrm{O} 303 \mu \mathrm{m}$ $2_{02} \rightarrow 1_{11}$ line. In this process we have also taken the best fit model for the inner component into account. The observed line ratios are best reproduced with a dust opacity in the range $\tau_{100}=0.25-1$. In this component collisional excitation becomes non-negligible for $n_{\mathrm{H}_{2}}>1 \times 10^{5} \mathrm{~cm}^{-3}$, but in these models the undetected $\mathrm{H}_{2} \mathrm{O} 269 \mu \mathrm{m} 1_{11} \rightarrow 0_{00}$ line goes into emission, 


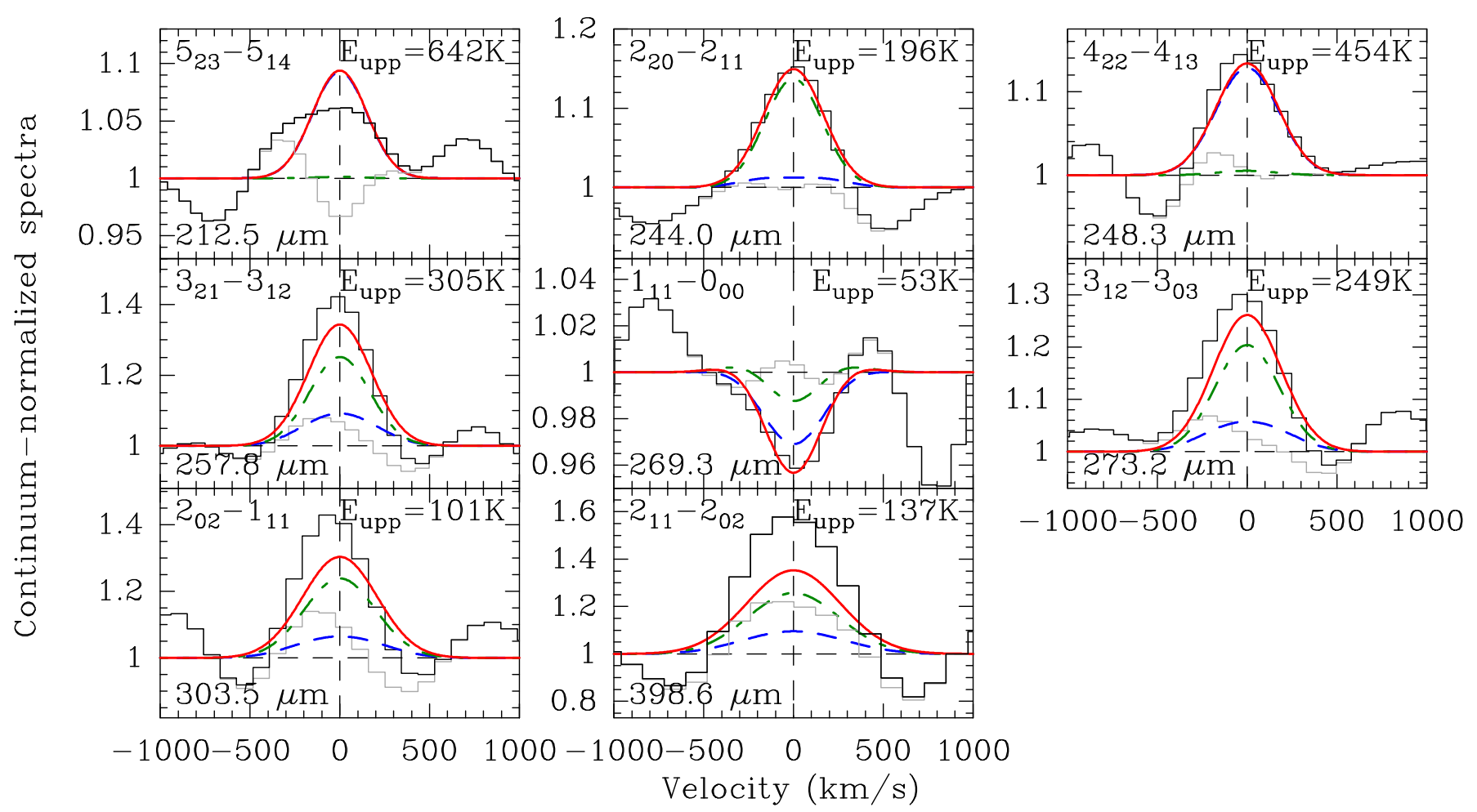

Fig. 6. $\mathrm{H}_{2} \mathrm{O}$ lines observed in Arp 299A with SPIRE. The black histograms are the observed, continuum-normalized, spectra. The best fit models from Sect. 3 are also included. The dashed blue and dashed-dotted green curves denote the models for the inner and outer components, respectively, and the solid red curve denotes the sum of the models. Gray histograms are the residual spectra after model subtraction.

Table 5. Parameters of the continuum models.

\begin{tabular}{ccccccc}
\hline \hline $\mathrm{C}^{a}$ & $\begin{array}{c}\text { Radius }^{b} \\
(\mathrm{pc})\end{array}$ & $\begin{array}{c}T_{\mathrm{d}}{ }^{b} \\
(\mathrm{~K})\end{array}$ & $\tau_{100^{b}}$ & $\begin{array}{c}N_{\mathrm{H}_{2}{ }^{c}} \\
\left(\mathrm{~cm}^{-2}\right)\end{array}$ & $\begin{array}{c}M^{d} \\
\left(10^{8} M_{\odot}\right)\end{array}$ & $\begin{array}{c}L^{e} \\
\left(L_{\odot}\right)\end{array}$ \\
\hline$C_{\text {inner }}$ & $20-25$ & $90-120$ & $1-4$ & $(1-3) \times 10^{24}$ & $0.25-1.25$ & $(0.7-1.5) \times 10^{11}$ \\
$C_{\text {outer }}$ & $50-100$ & $70-90$ & $0.25-1$ & $(1.5-7) \times 10^{23}$ & $0.25-5$ & $(1.7-3.0) \times 10^{11}$ \\
\hline
\end{tabular}

Notes. ${ }^{(a)}$ Component. ${ }^{(b)}$ Independent parameter. ${ }^{(c)}$ Column density of $\mathrm{H}_{2}$, calculated assuming a mass-absorption coefficient of $44.4 \mathrm{~cm}^{2} \mathrm{~g}^{-1}$ at $100 \mu \mathrm{m}$ and a gas-to-dust mass ratio of $100 .{ }^{(d)}$ Estimated gas mass, assuming spherical symmetry. ${ }^{(e)}$ Unattenuated dust luminosity of the component.

Table 6. Derived molecular column densities and abundances.

\begin{tabular}{lccccc}
\hline \hline $\mathrm{C}^{a}$ & $\begin{array}{c}N_{\mathrm{H}_{2} \mathrm{O}} / \tau_{50} b, c \\
\left(\mathrm{~cm}^{-2}\right)\end{array}$ & $\begin{array}{c}N_{\mathrm{OH}} / \tau_{50} b, c \\
\left(\mathrm{~cm}^{-2}\right)\end{array}$ & $\begin{array}{c}N_{{ }^{18} \mathrm{OH}} / \tau_{50} b, c \\
\left(\mathrm{~cm}^{-2}\right)\end{array}$ & $\chi_{\mathrm{H}_{2} \mathrm{O}}{ }^{d}$ & $\chi_{\mathrm{OH}}{ }^{d}$ \\
\hline$C_{\text {inner }}$ & $(0.5-2) \times 10^{18}$ & $(0.2-1) \times 10^{19}$ & $<5 \times 10^{15}$ & $(1-5) \times 10^{-6}$ & $(0.5-5) \times 10^{-5}$ \\
$C_{\text {outer }}$ & $(0.2-1) \times 10^{17}$ & $(2-8) \times 10^{17}$ & $<5 \times 10^{15}$ & $(0.5-2.5) \times 10^{-7}$ & $(0.5-1) \times 10^{-6}$ \\
\hline
\end{tabular}

Notes. ${ }^{(a)}$ Component. ${ }^{(b)}$ Independent parameter. ${ }^{(c)}$ Column density per unit of dust opacity at $50 \mu \mathrm{m}, \tau_{50}{ }^{(d)}$ Estimated molecular abundance relative to $\mathrm{H}$ nuclei, with an estimated column of $\mathrm{H}$ nuclei per $\tau_{50}$ of $4 \times 10^{23} \mathrm{~cm}^{-2}$.

favoring models with lower $n_{\mathrm{H}_{2}}$ where collisional excitation is negligible. The reduced $\chi^{2}$ for our grid of models at the preferred dust opacity $\tau_{100}=0.5$ is presented in Fig. 11. The line ratios are best reproduced with dust temperatures between $T_{\text {dust }}=70$ and $90 \mathrm{~K}$, and $\mathrm{H}_{2} \mathrm{O}$ columns between $N_{\mathrm{H}_{2} \mathrm{O}} / \tau_{50}=2 \times 10^{16}$ and $1 \times 10^{17} \mathrm{~cm}^{-2}$. To also account for the absolute fluxes, the component should have a radius of $R=50-100 \mathrm{pc}$. The best fit is achieved with $\tau_{100}=0.5, T_{\text {dust }}=70 \mathrm{~K}, N_{\mathrm{H}_{2} \mathrm{O}} / \tau_{50}=5 \times 10^{16} \mathrm{~cm}^{-2}$ and $R=75 \mathrm{pc}$.
In Fig. 12 we present the reduced $\chi^{2}$ for the $\mathrm{OH}$ doublets in the outer component for different values of $N_{\mathrm{OH}} / \tau_{50}$ applied to the best fit model found for $\mathrm{H}_{2} \mathrm{O}$, now taking the $\mathrm{OH}$ contribution from the inner component into account. The $\mathrm{OH} 119 \mu \mathrm{m}$ ${ }^{2} \Pi_{3 / 2}-{ }^{2} \Pi_{3 / 2} \frac{5}{2}-\frac{3}{2}$ doublet which is redshifted by $\sim 175 \mathrm{~km} \mathrm{~s}^{-1}$ is not included here. The best fit is achieved with a column density of $\mathrm{OH}$ in the range $N_{\mathrm{OH}} / \tau_{50}=2 \times 10^{17}$ to $8 \times 10^{17} \mathrm{~cm}^{-2}$, corresponding to an $\mathrm{OH} / \mathrm{H}_{2} \mathrm{O}$ ratio in the range 4 to 16 . Applying a similar process to the $3 \sigma$ upper limits of the ${ }^{18} \mathrm{OH}$ 


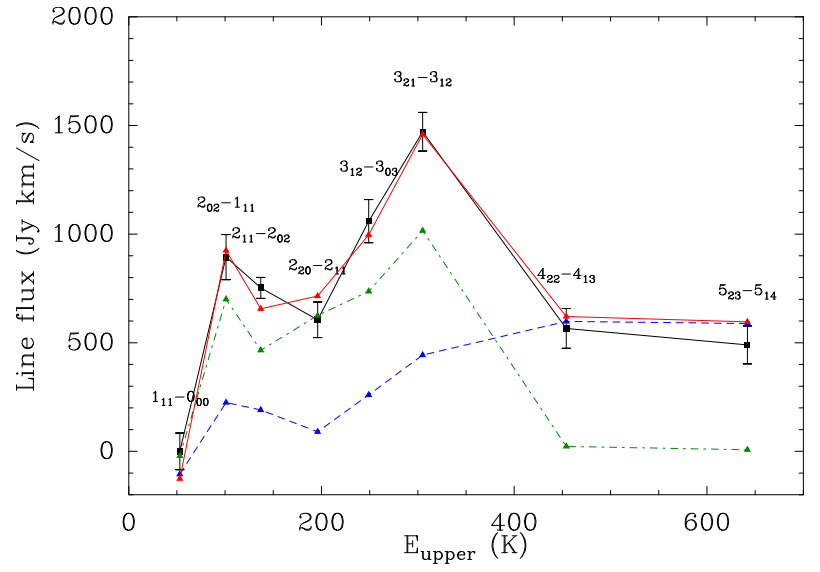

Fig. 7. Spectral line energy distribution of the $\mathrm{H}_{2} \mathrm{O}$ lines detected with SPIRE, the black squares represent the data. Model predictions are included with the dashed blue and dashed-dotted green curves denoting the models for the inner and outer components, respectively. The solid red curve is the sum of these two models.

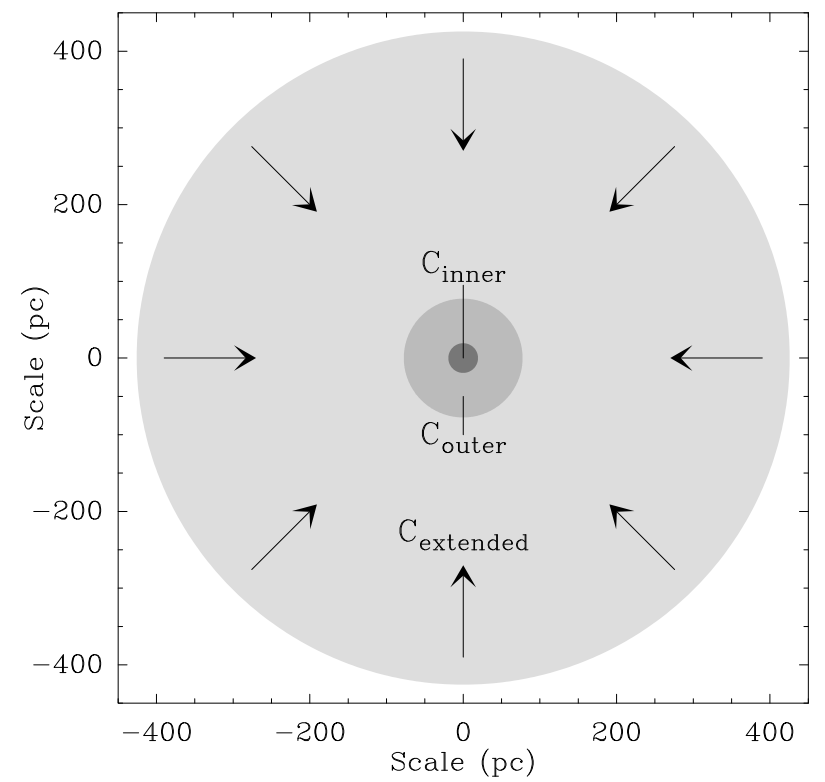

Fig. 8. Schematic representation of the spherically symmetric model for the molecular components of Arp 299A showing approximate spatial scales. Most of the molecular absorption is formed in the outer layers of the dense, most compact $\mathrm{C}_{\text {inner }}$ component while the $\mathrm{H}_{2} \mathrm{O}$ emission in the submillimeter seems to originate in the more optically thin $\mathrm{C}_{\text {outer }}$ component. A tentative inflowing $\mathrm{C}_{\text {extended }}$ component is included to account for the redshifted ground state $\mathrm{OH}$ doublet at $119 \mu \mathrm{m}$.

doublets we get an upper limit to the ${ }^{18} \mathrm{OH}$ column density of $N_{18} \mathrm{OH} / \tau_{50}<5 \times 10^{15} \mathrm{~cm}^{-2}$.

\subsection{Extended component}

Most of the flux in both the $\mathrm{H}_{2} \mathrm{O}$ and the $\mathrm{OH}$ lines is reproduced with the two components described in Sects. 3.2 and 3.3. The ground state $\mathrm{OH}^{2} \Pi_{3 / 2}-{ }^{2} \Pi_{3 / 2} \frac{5}{2}-\frac{3}{2}$ doublet at $119 \mu \mathrm{m}$ is, however, redshifted by $\sim 175 \mathrm{~km} \mathrm{~s}^{-1}$ compared with the models, and the continuum at wavelengths $\gtrsim 50 \mu \mathrm{m}$ is underpredicted by the combined central components. One way to shift the ground state $\mathrm{OH}$ doublet and simultaneously reproduce some of the continuum at longer wavelengths is to include a cooler and more extended component with a uniform inward velocity field. In this

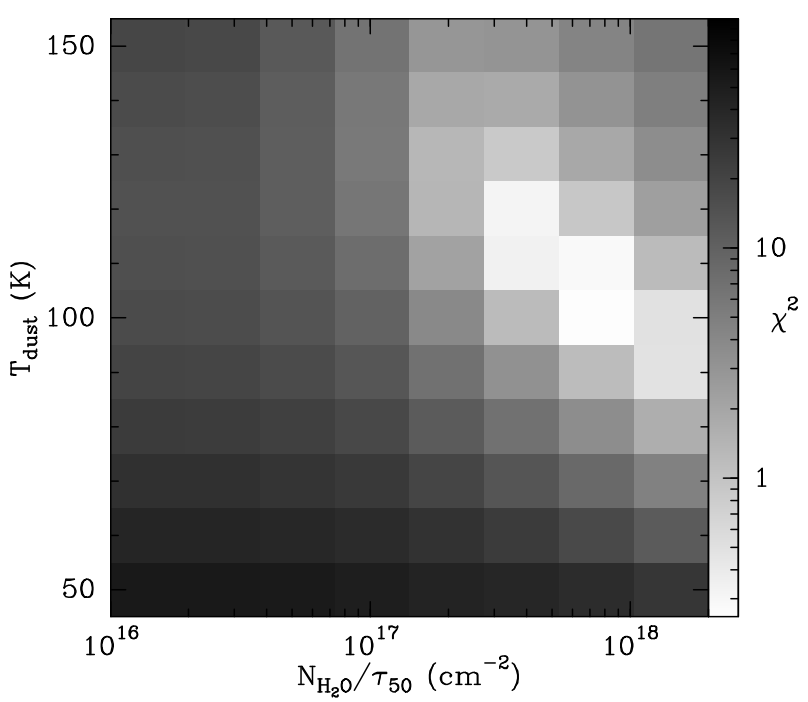

Fig. 9. Reduced $\chi^{2}$ for the observed high-lying $\mathrm{H}_{2} \mathrm{O}$ lines in our grid of models with $\tau_{100}=2$.

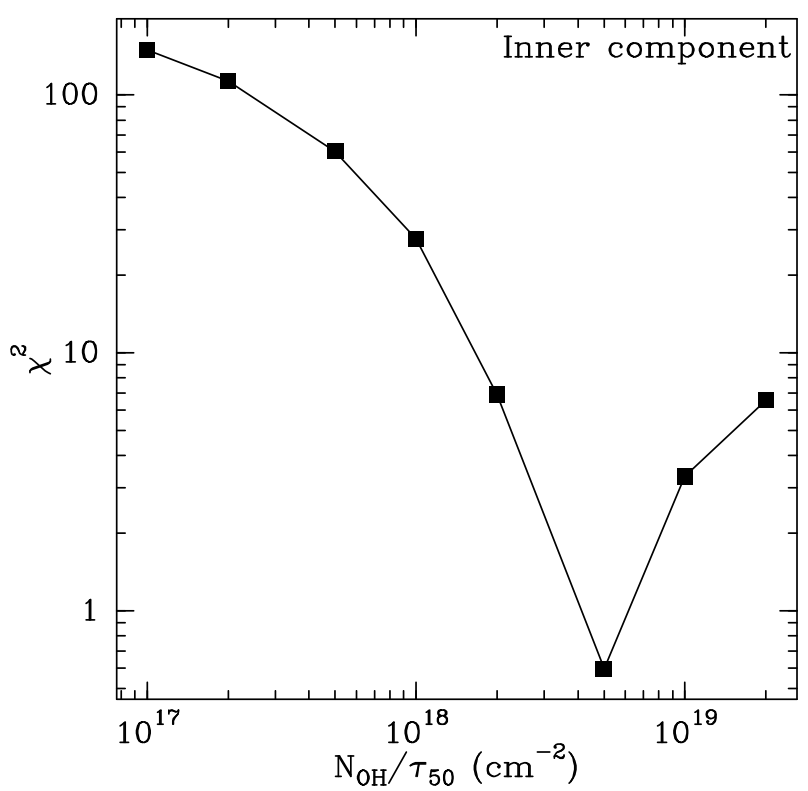

Fig. 10. $\chi^{2}$ for the $\mathrm{OH}^{2} \Pi_{1 / 2}-{ }^{2} \Pi_{1 / 2} \frac{7}{2}-\frac{5}{2}$ at $71 \mu \mathrm{m}$ with different values of $N_{\mathrm{OH}} / \tau_{50}$ applied to the best fit model of the inner component found using the $\mathrm{H}_{2} \mathrm{O}$ lines.

component the $\mathrm{OH}$ will mostly be in the ground state and produce redshifted absorption from the gas in front of the nucleus as well as emission at systemic velocity from gas on the sides, effectively shifting the ground state $\mathrm{OH}$ doublet to longer wavelengths while leaving the other transitions mostly unchanged.

Our limited angular resolution precludes an accurate quantitative analysis of the inflow energetics, but the lack of $\mathrm{OH} 119 \mu \mathrm{m}{ }^{2} \Pi_{3 / 2}-{ }^{2} \Pi_{3 / 2} \frac{5}{2}-\frac{3}{2}$ absorption at systemic velocities, where the $\mathrm{OH}$ columns are extremely large, suggests some cancellation between the expected absorption and emission at blueshifted velocities. This blueshifted emission is indeed seen in neighboring spaxels (Fig. 13), and the combined redshifted absorption and surrounding blueshifted emission are consistent with an extended inflow. The geometry of the inflowing gas may significantly depart from sphericity, and thus the values we give, based on our spherical radiative transfer approach, should be 


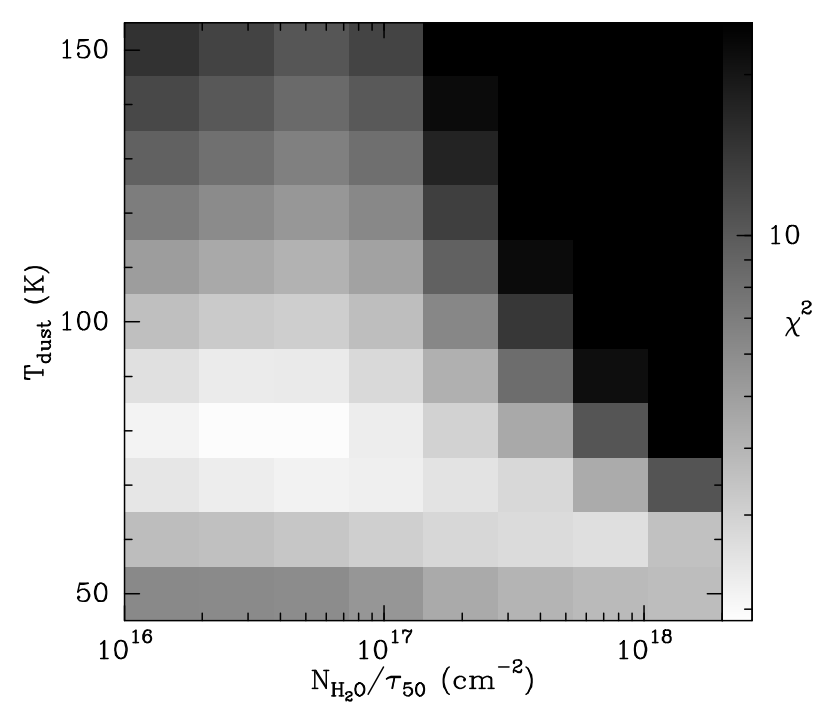

Fig. 11. Reduced $\chi^{2}$ for the observed low-lying $\mathrm{H}_{2} \mathrm{O}$ lines in our grid of models with $\tau_{100}=0.5$ for the outer component, taking the contribution from the inner component into account.

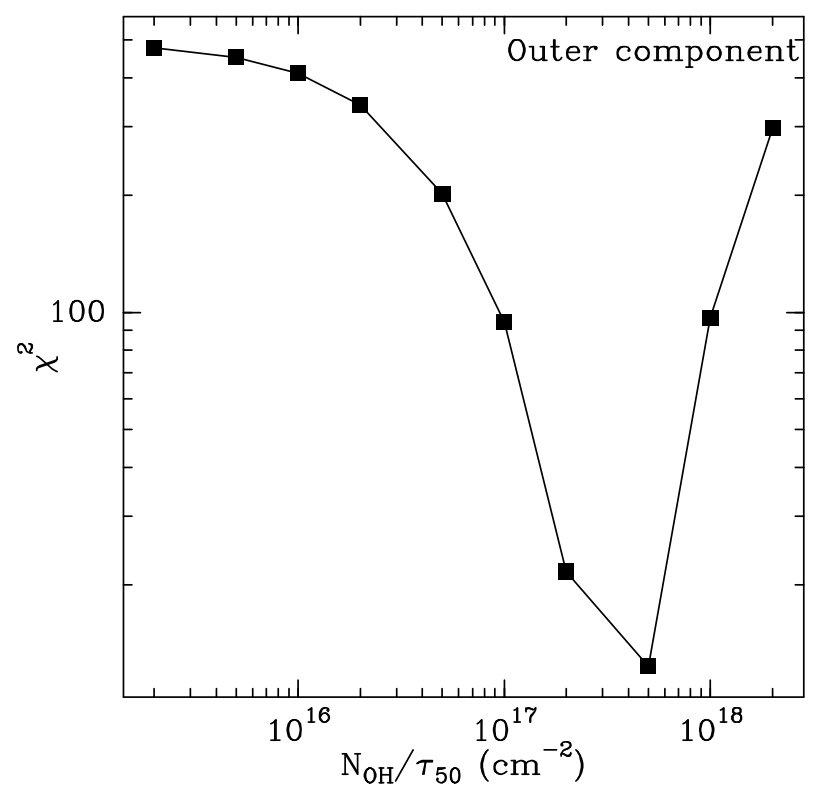

Fig. 12. Reduced $\chi^{2}$ for the $\mathrm{OH}$ doublets, except for $\mathrm{OH} 119 \mu \mathrm{m}$ ${ }^{2} \Pi_{3 / 2}-{ }^{2} \Pi_{3 / 2} \frac{5}{2}-\frac{3}{2}$, with different values of $N_{\mathrm{OH}} / \tau_{50}$ applied to the best fit model of the outer component found using the $\mathrm{H}_{2} \mathrm{O}$ lines.

considered a first approach; further high-angular observations will be required to better constrain the energetics.

In Fig. 3 as well as in the $\mathrm{OH}$ panels of Fig. 4 we have included an extended component with parameters $R=400 \mathrm{pc}$, $\tau_{100}=0.25, T_{\text {dust }}=400, N_{\mathrm{OH}} / \tau_{50}=4 \times 10^{15} \mathrm{~cm}^{-2}$ and a uniform velocity for the $\mathrm{OH}$ of $200 \mathrm{~km} \mathrm{~s}^{-1}$ towards the nucleus. Based on the total $\mathrm{OH}$ column and assuming a hemispherical inflow with the same $\mathrm{OH}$ abundance as in the outer component, a rough estimate of the mass inflow rate is $14-28 M_{\odot} \mathrm{yr}^{-1}$. With only one doublet available we do not attempt a full analysis of this component and the parameters are not well constrained, however the mass inflow rate we obtain is comparable to that inferred in NGC 4418 (G-A12; Costagliola et al. 2013; Sakamoto et al. 2013). We note that, in Fig. 3, the continuum of the combined components still significantly falls short of the observed data. Part of this discrepancy is likely the result of contamination from

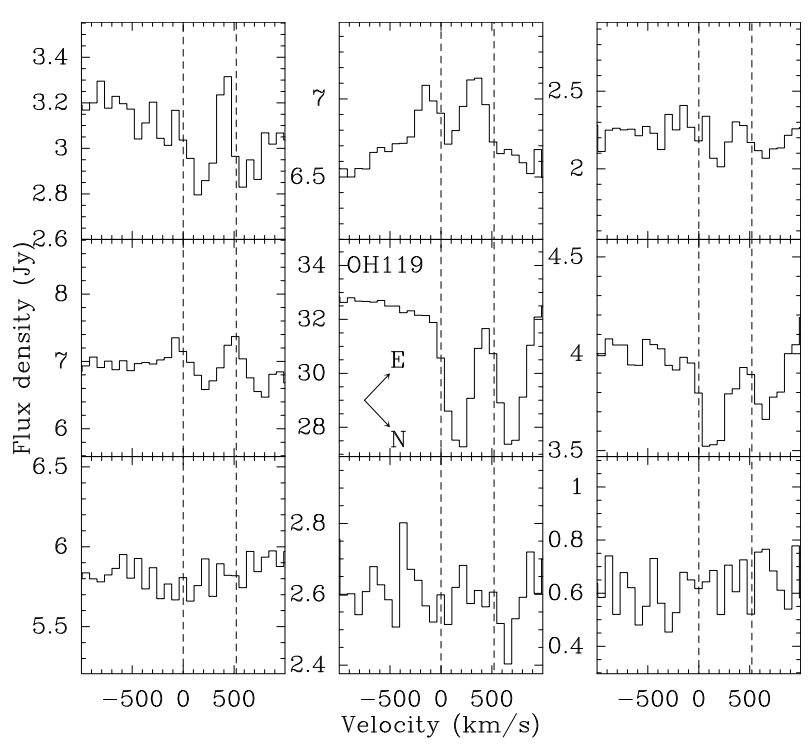

Fig. 13. Central nine spaxels in the observation of the ground state $\mathrm{OH}$ ${ }^{2} \Pi_{3 / 2}-{ }^{2} \Pi_{3 / 2} \frac{5}{2}-\frac{3}{2}$ doublet at $119 \mu \mathrm{m}$. The compass in the central spaxel shows the approximate orientation of the array on the sky. We note that, where detected, the absorption features are redshifted, and the emission features are blueshifted relative to the systemic velocity. Toward the southern spaxel (upper-left panel), both redshifted absorption and blueshifted emission are observed, showing an inverse P-Cygni profile.

the other two components of the system, but there are probably also regions of even more extended continuum emission, not associated with a molecular component.

\subsection{Underpredicted lines}

Even with three components included, not all data is well represented by our best-fit models. In the SPIRE range, the $\mathrm{H}_{2} \mathrm{O}$ $2_{11} \rightarrow 2_{02}$ line at $399 \mu \mathrm{m}$ (see last panel of Fig. 6) is underpredicted by our models. As noted in Sect. 2.1.2 this is the only SPIRE line that is detected with just the spectrometer long wavelength array, and due to the larger beam size it might be contaminated by flux from the other components of Arp 299. As a comparison, the $\mathrm{H}_{2} \mathrm{O} 2_{02} \rightarrow 1_{11}$ line at $303 \mu \mathrm{m}$, which is situated in the overlap region of the two SPIRE detectors, shows $\sim 30 \%$ more flux in the long wavelength array than in the short wavelength array.

In the PACS range, the $\mathrm{H}_{2} \mathrm{O} 4_{13} \rightarrow 4_{04}$ line at $187 \mu \mathrm{m}$ (see middle panel of last row in Fig. 4) is also clearly underpredicted by our models. A possible explanation is that the wavelength of this line places it in a transition region between the absorption lines, which are located at shorter wavelengths where the optical depth of the continuum is higher, and the emission lines, which are located at longer wavelengths where the continuum optical depth is lower. It is likely that such a line cannot be well predicted by our simple models, which use distinct, uniform components.

\section{Discussion}

\subsection{The gas and dust components of Arp $299 A$}

The properties of the two main components responsible for the molecular spectrum, described in Sect. 3, are summarized in Tables 5 and 6 . The components have sizes comparable to those of giant molecular clouds (GMCs) in the Galaxy but both 
are warmer as well as significantly more massive and luminous than typical GMCs, which have masses and luminosities of the order of $10^{5}-10^{6} M_{\odot}$ and $10^{5}-10^{8} L_{\odot}$, respectively (e.g., Scoville \& Good 1989).

With an estimated column density of $N_{\mathrm{H}_{2}} \sim 10^{24} \mathrm{~cm}^{-2}$ in the inner component, Arp 299A appears to be somewhat less obscured than the CONs in Arp 220, NGC 4418 and Zw 049.057 (G-A12; Falstad et al. 2015), but still close to the limit of being considered Compton-thick $\left(N_{\mathrm{H}} \gtrsim 1.5 \times 10^{24} \mathrm{~cm}^{-2}\right)$. Compared to these other CONs, the inner component of Arp 299A has many similarities with the warm component in NGC 4418 (G-A12) and also to the core component of $\mathrm{Zw} 049.057$ (Falstad et al. 2015). The outer component of Arp 299A is more similar to the extended component of NGC 4418 (G-A12) or to the outer component of Zw 049.057 (Falstad et al. 2015), which is, however, estimated to be cooler than that of Arp 299A.

As in $\mathrm{Zw} 049.057$ and NGC 4418, the central regions of Arp 299A are extreme compared to the center of the Milky Way, where a comparable amount of molecular gas (few $\times$ $\left.10^{7} M_{\odot}\right)$ is distributed on much larger $(200-500$ pc) scales (e.g., Cox \& Laureijs 1989; Morris \& Serabyn 1996; Ferrière et al. 2007). Our results show that galaxies with luminous [C II] may also have hidden CONs, and thus have nuclei with very young, compact, and embedded activity.

\subsubsection{Inflowing gas in the nucleus?}

The presence of gas flowing towards the nucleus is suggested by the redshift of $\sim 175 \mathrm{~km} \mathrm{~s}^{-1}$ in the $\mathrm{OH}$ ground state ${ }^{2} \Pi_{3 / 2}-{ }^{2} \Pi_{3 / 2} \frac{5}{2}-\frac{3}{2}$ doublet at $119 \mu \mathrm{m}$. An alternative explanation for this feature could be that it is formed in low-excited gas in the foreground, not directly associated with the central regions of Arp 299A. However, similar, but weaker and less redshifted features are seen in some of the neighboring PACS spaxels along with weak blueshifted emission, indicating that the feature is indeed associated with the nucleus. As the gas seems to be inflowing on spatial scales larger than the two central components, it is not as highly excited and we only see it in the ground state $\mathrm{OH}$ transition. Based on our rudimentary modeling of the redshifted doublet (see Sect. 3.4), assuming a hemispherical inflow, we estimate an upper limit to the mass inflow rate of 14-28 $M_{\odot} \mathrm{yr}^{-1}$. This molecular inflow could provide the nucleus with fresh material.

\subsubsection{Molecular abundances}

We find high abundances, relative to $\mathrm{H}$ nuclei, of $\mathrm{H}_{2} \mathrm{O}$ and $\mathrm{OH}$ in the inner component, $1-5 \times 10^{-6}$ and $0.5-5 \times 10^{-5}$, respectively. The $\mathrm{H}_{2} \mathrm{O}$ and $\mathrm{OH}$ abundances found in the outer component are $0.5-2.5 \times 10^{-7}$ and $0.5-1 \times 10^{-6}$, respectively. We thus see high $\mathrm{OH} / \mathrm{H}_{2} \mathrm{O}$ ratios of $\gtrsim 1$ in both components.

The $\mathrm{H}_{2} \mathrm{O}$ abundances in the inner component are comparable to those found in the eastern nucleus of Arp 220 (G-A12) as well as in $\mathrm{Zw} 049.057$ (Falstad et al. 2015). In the Galaxy, there are no regions of comparable size with $\mathrm{H}_{2} \mathrm{O}$ abundances this high, but on smaller scales, hot cores (e.g., in NGC 6334 I and Orion KL; Emprechtinger et al. 2013; Melnick et al. 2010) can have abundances as high as $10^{-5}$. In the outer component, the $\mathrm{H}_{2} \mathrm{O}$ abundances are similar to those found in the warm component of NGC 4418 (G-A12). The $\mathrm{OH}$ abundances in the inner component are more similar to those in the western nucleus of Arp 220 and the core component of NGC 4418 (G-A12). In the Galaxy, a similar $\mathrm{OH}$ abundance was found in the shocked low-velocity gas around the compact infrared source Orion-IRc2 by Wright et al. (2000).

To account for the high $\mathrm{H}_{2} \mathrm{O}$ and $\mathrm{OH}$ abundances, we consider three main scenarios: ion-neutral reactions initialized by cosmic-ray or X-ray ionization of $\mathrm{H}_{2}$ (van Dishoeck et al. 2013), sublimation of $\mathrm{H}_{2} \mathrm{O}$ ice from dust grains when the dust temperature rises above $\sim 100 \mathrm{~K}$ (Fraser et al. 2001; van Dishoeck et al. $2013)$, and neutral-neutral reactions in warm gas $(T \gtrsim 250 \mathrm{~K})$ (Neufeld et al. 1995; van Dishoeck et al. 2013). If the high abundances are due to sublimation from dust-grains, they will eventually decrease unless the core is replenished with icy dust grains by the inflow or there is another formation process to efficiently counter $\mathrm{H}_{2} \mathrm{O}$ and $\mathrm{OH}$ destruction by, for example, photodissociation. In the other two scenarios, the abundances can persist as long as the formation conditions stay the same.

In the inner component, where the highest abundances are found, the dust temperature is $90-120 \mathrm{~K}$, enough for sublimation to occur. As we are unable to put any constraints on the gas temperature, we cannot rule out neutral-neutral reactions in warm gas. In the ion-neutral scenario, $\mathrm{H}_{2} \mathrm{O}$ and $\mathrm{OH}$ are formed through dissociative recombination of $\mathrm{H}_{3} \mathrm{O}^{+}$with a branching ratio that favors $\mathrm{OH}$ (Herd et al. 1990; Jensen et al. 2000). Although we do not exclude the possibility of contributions from the other two processes, the fact that we see high $(>1) \mathrm{OH} / \mathrm{H}_{2} \mathrm{O}$ abundance ratios in both components hints at ion-neutral reactions playing an important part. Models of dense molecular gas irradiated by high rates of cosmic-rays or X-rays predict $\mathrm{OH} / \mathrm{H}_{2} \mathrm{O}$ ratios that can go above unity, with abundances that are consistent with our results (Maloney et al. 1996; Meijerink \& Spaans 2005; Meijerink et al. 2011).

We note that the highest-lying $\mathrm{OH}$ doublets, $\mathrm{OH}$ ${ }^{2} \Pi_{1 / 2}-{ }^{2} \Pi_{1 / 2} \frac{7}{2}-\frac{5}{2}$ and ${ }^{2} \Pi_{3 / 2}-{ }^{2} \Pi_{3 / 2} \frac{9}{2}-\frac{7}{2}$ at 71 and $65 \mu \mathrm{m}$, in Arp 299A have higher strengths, relative to lower-lying $\mathrm{OH}$ doublets and higher-lying $\mathrm{H}_{2} \mathrm{O}$ lines, than in Arp 220, Zw 049.057, or even NGC 4418 (G-A12; Falstad et al. 2015). This explains the very high abundance we infer for $\mathrm{OH}$, compared to the other galaxies.

\subsection{Evolutionary stage - the ${ }^{16} \mathrm{O} /{ }^{18} \mathrm{O}$ ratio}

The secondary nuclide ${ }^{18} \mathrm{O}$ is produced in massive stars and is expected to increase in relation to the primary nuclide ${ }^{16} \mathrm{O}$ with each new generation of stars (Prantzos et al. 1996). The abundance ratio of ${ }^{16} \mathrm{O}$ to ${ }^{18} \mathrm{O}$ can therefore reflect the initial mass function (IMF) as well as the amount of stellar processing that the gas has gone through so far. A potential problem is that, under certain conditions, the small difference in molecular binding energies might cause the oxygen isotopes to be unequally distributed among molecules. Langer et al. (1984) found that such chemical fractionation effects for oxygen isotopes are small under all conditions, and our results for $\mathrm{OH}$ can be used directly to estimate the ${ }^{16} \mathrm{O} /{ }^{18} \mathrm{O}$ ratio.

González-Alfonso et al. (2012) use the ${ }^{16} \mathrm{O} /{ }^{18} \mathrm{O}$ ratio as part of a tentative evolutionary scenario in which Mrk 231 with its extreme ${ }^{16} \mathrm{O} /{ }^{18} \mathrm{O}$ ratio of $\gtrsim 30$ (Fischer et al. 2010; González-Alfonso et al. 2014b) has a more evolved starburst than Arp 220 (G-A12) and NGC 4418 which have ratios of $\sim 70$ and $\sim 500$, respectively (G-A12). In this scenario $\mathrm{Zw} 049.057$, with a ratio of 50-100 (Falstad et al. 2015), can be placed at a similar evolutionary stage as Arp 220. This of course assumes that the starbursts in these sources are of comparable sizes in relation to the host galaxies, that they have similar IMF and mixing of the ISM, and that the models are correct. 
We do not detect any transitions of the ${ }^{18} \mathrm{O}$ isotopologues of $\mathrm{OH}$ or $\mathrm{H}_{2} \mathrm{O}$ in Arp 299A, but from the upper limits of the observed ${ }^{18} \mathrm{OH}$ lines we estimate a lower limit of the ${ }^{16} \mathrm{O} /{ }^{18} \mathrm{O}$ ratio of 400 in the inner component. In the outer component we find a similar upper limit of the ${ }^{18} \mathrm{OH}$ column, but due to the lower estimated ${ }^{16} \mathrm{OH}$ column in this component, we only get a lower limit of the ${ }^{16} \mathrm{O} /{ }^{18} \mathrm{O}$ ratio of 40 . In the case of the undetected $\mathrm{H}_{2}^{18} \mathrm{O}$ lines, the corresponding $\mathrm{H}_{2}^{16} \mathrm{O}$ lines are not strong and an upper limit of the $\mathrm{H}_{2}^{18} \mathrm{O}$ column would be close to our estimate for the $\mathrm{H}_{2}^{16} \mathrm{O}$ column. A lower limit of the ${ }^{16} \mathrm{O} /{ }^{18} \mathrm{O}$ ratio estimated from the undetected $\mathrm{H}_{2}^{18} \mathrm{O}$ transitions would therefore be low and not very meaningful. While the inner component with its ratio of $>400$, reminiscent of NGC 4418, shows no signs of strong stellar processing, we cannot rule out the presence of an evolved starburst in the outer component, which has a lower limit that would put it close to Mrk 231 on the evolutionary sequence.

\subsubsection{What governs the ${ }^{16} \mathrm{O} /{ }^{18} \mathrm{O}$ ratio?}

Another interesting similarity between the "young" NGC 4418 and Arp 299A is the fact that they both show signs of molecular gas inflowing onto the nucleus but lack outflow signatures. NGC 4418 exhibits redshifted absorption by $\sim 100 \mathrm{~km} \mathrm{~s}^{-1}$ in the [O I] $63 \mu \mathrm{m}$ line and the ground state $\mathrm{OH}^{2} \Pi_{3 / 2}-{ }^{2} \Pi_{3 / 2} \frac{5}{2}-\frac{3}{2}$ doublet at $119 \mu \mathrm{m}$ (G-A12) while the same ground state doublet is redshifted by $175 \mathrm{~km} \mathrm{~s}^{-1}$ in Arp 299A (see Sect. 4.1.1). Together with the higher ${ }^{16} \mathrm{O} /{ }^{18} \mathrm{O}$ ratio, this might indicate that the nuclei of both galaxies are in an earlier stage of evolution, before the onset of mechanical feedback. If this is the case, the ratio can be used as a tool to estimate the age of any starburst activity as it should decrease with every new generation of stars. Of course, this will also depend on how much of the gas reservoir is used by the starburst and how much remains unused. The high ratio could also simply mean that the molecular gas reservoirs in Arp 299A and NGC 4418 are replenished with relatively unprocessed gas, thus keeping the ${ }^{16} \mathrm{O} /{ }^{18} \mathrm{O}$ ratio higher than in galaxies without inflows of molecular gas. A similar scenario seems to apply for the merger NGC 1614 (König et al. 2016).

\subsection{The nuclear power source}

The surface brightness of the inner component is high, $\sim 5 \times$ $10^{13} L_{\odot} \mathrm{kpc}^{-2}$ over $50 \mathrm{pc}$, but its Compton-thick nature makes it difficult to unambiguously identify the embedded power source. Although this surface brightness is high for dusty radiationpressure-supported starbursts (Thompson et al. 2005) under normal conditions, it is still well below the theoretical value of $\sim 10^{15} L_{\odot} \mathrm{kpc}^{-2}$ that can be attained in hot $\left(T_{\mathrm{d}}>200 \mathrm{~K}\right)$ starbursts (Andrews \& Thompson 2011). Such dust temperatures are not seen in our models, but due to the high obscuration, we cannot rule out their existence.

Fischer et al. (2014) used the Cloudy (Ferland et al. 2013) spectral synthesis code to create one-dimensional models of a gas and dust cloud, centrally illuminated by either a starburst or an AGN. They explored hydrogen column densities, $N_{\mathrm{H}}$, up to $10^{25} \mathrm{~cm}^{-2}$ and ionization parameters, $U$, between $10^{-4}$ and 1 for hydrogen densities at the illuminated face of the cloud, $n_{\mathrm{H}^{+}}$, of 30,300 and $3000 \mathrm{~cm}^{-3}$. In these idealized models, a central starburst with $n_{\mathrm{H}^{+}}=30 \mathrm{~cm}^{-3}$ can, in principle, reproduce the high $\mathrm{OH}$ column densities and high $\mathrm{OH} / \mathrm{H}_{2} \mathrm{O}$ ratios that we find in Arp 299A. The higher density starburst models, however, underpredict both the column density of $\mathrm{OH}$ and the $\mathrm{OH} / \mathrm{H}_{2} \mathrm{O}$ ratio. The AGN models on the other hand are able to produce higher
$\mathrm{OH}$ column densities and $\mathrm{OH} / \mathrm{H}_{2} \mathrm{O}$ ratios for all three values of $n_{\mathrm{H}^{+}}$, resulting in better agreement with our results in Arp 299A for a wide range of parameters. These high rates of $\mathrm{OH} / \mathrm{H}_{2} \mathrm{O}$ are due to X-rays due to the AGN. Another possibility, not included in their starburst models, is that high rates of cosmic-ray ionization drive the chemistry. We note that these models assume a single, centrally concentrated, radiation source. Thus, we cannot rule out a distributed starburst or a combination of an AGN and a starburst.

Based on very long baseline interferometry (VLBI) radio observations, Pérez-Torres et al. (2010) concluded that there is a low-luminosity AGN in the central regions of Arp 299A. The bolometric luminosity of this putative AGN was estimated by Alonso-Herrero et al. (2013) to be $\sim 1.6 \times 10^{10} L_{\odot}$, corresponding to $10-20 \%$ of the IR luminosity of the inner component in our models and thus not enough to be the dominant power source. The presence of a nuclear starburst in Arp 299A is supported by the many supernovae and supernova remnants detected in the central $\sim 100$ pc using VLBI (e.g., Neff et al. 2004; Ulvestad 2009; Pérez-Torres et al. 2009). Herrero-Illana et al. (2012) analyzed the radial distribution of supernovae in the nuclear starburst of Arp 299A and found it to be consistent with an exponential disk with a scale length of 20-30 pc around the low-luminosity AGN reported by Pérez-Torres et al. (2010). The spatial scale of this star formation indicates that it might be associated with the inner component of our models.

\subsection{Is there an outflow?}

Signatures of molecular outflows have been observed in other CON galaxies (e.g., Arp 220, NGC 1377, and Zw 049.057; GA12; Aalto et al. 2012; Falstad et al. 2015). We do not see an outflow in Arp 299A, but a possible signature is the blueshifted $\mathrm{H}_{2} \mathrm{O}$ maser which Tarchi et al. (2011) associate with the expanding structure first suggested by Baan \& Haschick (1990). In NGC 3256, which, like Arp 299A, is a merger LIRG, Sakamoto et al. (2014) detected an outflow with a maximum velocity of $>750 \mathrm{~km} \mathrm{~s}^{-1}$ and a mass outflow rate of $>60 M_{\odot} \mathrm{yr}^{-1}$. Furthermore, $\mathrm{OH}$ observations have revealed massive molecular outflows in a number of ULIRGs (e.g., Fischer et al. 2010; Sturm et al. 2011) and in systematic searches Veilleux et al. (2013) and Spoon et al. (2013) found evidence of high velocity outflows in approximately two thirds of their (U)LIRG/QSO and ULIRG samples, respectively. We note that the processes responsible for outflows in less luminous galaxies may be very different from those of the most powerful outflows, which are found in ULIRGs and galaxies hosting AGNs (see also Cicone et al. 2014). So why do we not see an outflow in Arp 299A?

With a luminosity of $\sim 1.6 \times 10^{10} L_{\odot}$, estimated by (Alonso-Herrero et al. 2013), and assuming a velocity of $500 \mathrm{~km} \mathrm{~s}^{-1}$, radiation pressure from the AGN should be able to drive an outflow of $\sim 0.6 M_{\odot} \mathrm{yr}^{-1}$. Bondi et al. (2012) estimated a lower limit to the core-collapse supernova rate in Arp 299A of $v_{\mathrm{SN}}>0.8 \mathrm{yr}^{-1}$. Using this lower limit in Eqs. (10) and (34) in Murray et al. (2005), and assuming that $10 \%$ of the energy is transferred to the ISM, we find that the supernovae could potentially drive a $500 \mathrm{~km} \mathrm{~s}^{-1}$ outflow of $32-50 M_{\odot} \mathrm{yr}^{-1}$. Assuming an age of $10 \mathrm{Myr}$, the total outflow mass would then be $6 \times 10^{6}-5 \times 10^{8} M_{\odot}$. If outflowing directly towards us, an outflow of this magnitude should be clearly detectable in absorption towards the continuum source. However, if oriented differently, projection effects or obscuration from the galaxy could make an outflow hard to detect. For example, with the assumed velocity of $500 \mathrm{~km} \mathrm{~s}^{-1}$ and a $45^{\circ}$ inclination, a given parcel of 
outflowing gas could occult the $<100 \mathrm{pc}$ outer component for up to $\sim 3 \times 10^{5} \mathrm{yr}$, reducing the possible amount of outflowing gas in front of the continuum source to $1.8 \times 10^{5}-1.5 \times 10^{7} M_{\odot}$.

\subsection{Outlook}

The surface brightness of the inner component is consistent with either an AGN, a nuclear starburst, or a combination of the two, as the source(s) of its luminosity. An obscured AGN could also explain the high $\mathrm{OH} / \mathrm{H}_{2} \mathrm{O}$ ratios seen in both the inner and outer components, with the luminosity estimated by Alonso-Herrero et al. $(2013)\left(\sim 1.6 \times 10^{10} L_{\odot}\right)$ it would account for up to $20 \%$ of the IR luminosity from the inner component. As there is also evidence for starburst activity at the scale of our inner component (Herrero-Illana et al. 2012), we deem it most likely that a composite AGN/starburst is responsible for the high luminosity emanating from the central regions of Arp 299A. This nuclear activity could be fed by the infalling gas, provided that there is some process present to transport the gas all the way down to the central tens of parsecs of the nucleus. If this is the case, the replenishment of unprocessed gas might also explain the fact that the ${ }^{16} \mathrm{O} /{ }^{18} \mathrm{O}$ ratio is higher than in similar galaxies, despite the presence of a nuclear starburst. Further study of this inflowing component and how it relates to the inner regions of Arp 299A could shed some light on how the nuclear gas concentrations in CONs are assembled and how their activity is sustained.

\section{Conclusions}

A number of basic results were obtained from the spectroscopic observations of Arp 299A presented in this paper. The PACS spectroscopy revealed high excitation in $\mathrm{H}_{2} \mathrm{O}$ and $\mathrm{OH}$. A total of nine $\mathrm{H}_{2} \mathrm{O}$ lines and four $\mathrm{OH}$ doublets with lower level energies up to $E_{\text {lower }} \sim 600 \mathrm{~K}$ and $E_{\text {lower }} \sim 400 \mathrm{~K}$, respectively, were detected in absorption. In addition, one $\mathrm{H}_{2} \mathrm{O}$ line and one $\mathrm{OH}$ doublet were detected in emission. The ground state $\mathrm{OH}^{2} \Pi_{3 / 2}-{ }^{2} \Pi_{3 / 2} \frac{5}{2}-\frac{3}{2}$ doublet at $119 \mu \mathrm{m}$ is redshifted by $\sim 175 \mathrm{~km} \mathrm{~s}^{-1}$ and is the only line with significant emission outside of the central spaxel of PACS, indicating an inflow of lowexcited molecular gas. The SPIRE spectroscopy shows seven submillimeter $\mathrm{H}_{2} \mathrm{O}$ emission lines with upper state energies up to $E_{\text {upper }} \sim 650 \mathrm{~K}$, the ground state $\mathrm{H}_{2} \mathrm{O} 269 \mu \mathrm{m} 1_{11} \rightarrow 0_{00}$ line was however not detected. No ${ }^{18} \mathrm{OH}$ or $\mathrm{H}_{2}^{18} \mathrm{O}$ lines were detected with any of the instruments.

The observed lines in the absorption dominated PACS spectra and the emission dominated SPIRE spectrum as well as the continuum levels have been analyzed using multicomponent radiative transfer modeling. The inner component in our model has a high $\mathrm{H}_{2}$ column density $\left((1-3) \times 10^{24} \mathrm{~cm}^{-2}\right)$, indicating that it is Compton-thick. The dust temperature in this component is estimated to be $T_{\mathrm{d}}=90-120 \mathrm{~K}$. In addition, it has high $\mathrm{OH}$ column densities per unit of continuum optical depth at $50 \mu \mathrm{m}\left((0.2-2) 10^{19} \mathrm{~cm}^{-2}\right)$ and a high $\mathrm{OH} / \mathrm{H}_{2} \mathrm{O}$ ratio $>1$, indicating that ion-neutral chemistry induced by $\mathrm{X}$-rays or cosmicrays is important in the nucleus of Arp 299A. A high surface brightness, $\sim 5 \times 10^{13} L_{\odot} \mathrm{kpc}^{-2}$ on a scale of $\sim 50 \mathrm{pc}$, in the inner component indicates that the luminosity is powered by a buried AGN and/or a nuclear starburst. Our analysis indicates that a composite source is the most likely explanation. Finally, the non-detection of ${ }^{18} \mathrm{OH}$ suggests $a{ }^{16} \mathrm{O} /{ }^{18} \mathrm{O}$ ratio of $>400$ in the core of Arp 299A. This is similar to the ratio found in NGC 4418, and might indicate either that the starbursts of
Arp 299A and NGC 4418 are in an early evolutionary stage or that their gas reservoirs are replenished with relatively unprocessed gas through molecular inflows.

Acknowledgements. We thank the anonymous referee for a thorough and constructive report that helped improve the paper. N.F. and S.A. thank the Swedish National Space Board for generous grant support (grant numbers 145/11:1B, 285/12 and 145/11:1-3). E.G.-A. is a Research Associate at the HarvardSmithsonian Center for Astrophysics, and thanks the Spanish Ministerio de Economía y Competitividad for support under project FIS2012-39162-C06-01 and ESP2015-65597-C4-1-R, and NASA grant ADAP NNX15AE56G. Basic research in IR astronomy at NRL is funded by the US-ONR; J.F. acknowledges support from NHSC/JPL subcontracts 1435724 and 1456609. PACS has been developed by a consortium of institutes led by MPE (Germany) and including UVIE (Austria); KU Leuven, CSL, IMEC (Belgium); CEA, LAM (France); MPIA (Germany); INAFIFSI/OAA/OAP/OAT, LENS, SISSA (Italy); IAC (Spain). This development has been supported by the funding agencies BMVIT (Austria), ESA-PRODEX (Belgium), CEA/CNES (France), DLR (Germany), ASI/INAF (Italy), and CICYT/MCYT (Spain). SPIRE has been developed by a consortium of institutes led by Cardiff University (UK) and including Univ. Lethbridge (Canada); NAOC (China); CEA, LAM (France); IFSI, Univ. Padua (Italy); IAC (Spain); Stockholm Observatory (Sweden); Imperial College London, RAL, UCL-MSSL, UKATC, Univ. Sussex (UK); and Caltech, JPL, NHSC, Univ. Colorado (USA). This development has been supported by national funding agencies: CSA (Canada); NAOC (China); CEA, CNES, CNRS (France); ASI (Italy); MCINN (Spain); SNSB (Sweden); STFC, UKSA (UK); and NASA (USA). This research has made use of NASA's Astrophysics Data System (ADS) and of GILDAS software (http: //www . iram. fr/ IRAMFR/GILDAS)

\section{References}

Aalto, S., Radford, S. J. E., Scoville, N. Z., \& Sargent, A. I. 1997, ApJ, 475, L107

Aalto, S., Muller, S., Sakamoto, K., et al. 2012, A\&A, 546, A68

Alonso-Herrero, A., Rieke, G. H., Rieke, M. J., \& Scoville, N. Z. 2000, ApJ, 532,845

Alonso-Herrero, A., Rieke, G. H., Colina, L., et al. 2009, ApJ, 697, 660

Alonso-Herrero, A., Roche, P. F., Esquej, P., et al. 2013, ApJ, 779, L14

Andrews, B. H., \& Thompson, T. A. 2011, ApJ, 727, 97

Baan, W. A. 1985, Nature, 315, 26

Baan, W. A., \& Haschick, A. 1990, ApJ, 364, 65

Ballo, L., Braito, V., Della Ceca, R., et al. 2004, ApJ, 600, 634

Bondi, M., Pérez-Torres, M. A., Herrero-Illana, R., \& Alberdi, A. 2012, A\&A, 539, A134

Bradford, C. M., Bolatto, A. D., Maloney, P. R., et al. 2011, ApJ, 741, L37

Charmandaris, V., Stacey, G. J., \& Gull, G. 2002, ApJ, 571, 282

Cicone, C., Maiolino, R., Sturm, E., et al. 2014, A\&A, 562, A2

Costagliola, F., \& Aalto, S. 2010, A\&A, 515, A71

Costagliola, F., Aalto, S., Sakamoto, K., et al. 2013, A\&A, 556, A66

Cox, P., \& Laureijs, R. 1989, in The Center of the Galaxy, ed. M. Morris, IAU Symp., 136, 121

Daniel, F., Dubernet, M.-L., \& Grosjean, A. 2011, A\&A, 536, A76

Della Ceca, R., Ballo, L., Tavecchio, F., et al. 2002, ApJ, 581, L9

Dubernet, M.-L., Daniel, F., Grosjean, A., \& Lin, C. Y. 2009, A\&A, 497, 911

Emprechtinger, M., Lis, D. C., Rolffs, R., et al. 2013, ApJ, 765, 61

Falstad, N., González-Alfonso, E., Aalto, S., et al. 2015, A\&A, 580, A52

Ferland, G. J., Porter, R. L., van Hoof, P. A. M., et al. 2013, Rev. Mex. Astron. Astrofis., 49, 137

Ferrière, K., Gillard, W., \& Jean, P. 2007, A\&A, 467, 611

Fischer, J., Sturm, E., González-Alfonso, E., et al. 2010, A\&A, 518, L41 Fischer, J., Abel, N. P., González-Alfonso, E., et al. 2014, ApJ, 795, 117

Fixsen, D. J., Cheng, E. S., Gales, J. M., et al. 1996, ApJ, 473, 576

Fraser, H. J., Collings, M. P., McCoustra, M. R. S., \& Williams, D. A. 2001, MNRAS, 327, 1165

Gallais, P., Charmandaris, V., Le Floc'h, E., et al. 2004, A\&A, 414, 845

Gehrz, R. D., Sramek, R. A., \& Weedman, D. W. 1983, ApJ, 267, 551

González-Alfonso, E., \& Cernicharo, J. 1997, A\&A, 322, 938

González-Alfonso, E., \& Cernicharo, J. 1999, ApJ, 525, 845

González-Alfonso, E., Smith, H. A., Fischer, J., \& Cernicharo, J. 2004, ApJ, 613, 247

González-Alfonso, E., Smith, H. A., Ashby, M. L. N., et al. 2008, ApJ, 675, 303

González-Alfonso, E., Fischer, J., Isaak, K., et al. 2010, A\&A, 518, L43

González-Alfonso, E., Fischer, J., Graciá-Carpio, J., et al. 2012, A\&A, 541, A4 (G-A12) 
González-Alfonso, E., Fischer, J., Aalto, S., \& Falstad, N. 2014a, A\&A, 567, A91

González-Alfonso, E., Fischer, J., Graciá-Carpio, J., et al. 2014b, A\&A, 561, A27

González-Alfonso, E., Fischer, J., Sturm, E., et al. 2015, ApJ, 800, 69

Griffin, M. J., Abergel, A., Abreu, A., et al. 2010, A\&A, 518, L3

Henkel, C., Peck, A. B., Tarchi, A., et al. 2005, A\&A, 436, 75

Herd, C. R., Adams, N. G., \& Smith, D. 1990, ApJ, 349, 388

Herrero-Illana, R., Pérez-Torres, M. Á., \& Alberdi, A. 2012, A\&A, 540, L5

Impellizzeri, C. M. V., McKean, J. P., Castangia, P., et al. 2008, Nature, 456, 927

Jensen, M. J., Bilodeau, R. C., Safvan, C. P., et al. 2000, ApJ, 543, 764

König, S., Aalto, S., Muller, S., et al. 2016, A\&A, 594, A70

Langer, W. D., Graedel, T. E., Frerking, M. A., \& Armentrout, P. B. 1984, ApJ, 277,581

Maloney, P. R., Hollenbach, D. J., \& Tielens, A. G. G. M. 1996, ApJ, 466, 561

Meijerink, R., \& Spaans, M. 2005, A\&A, 436, 397

Meijerink, R., Spaans, M., Loenen, A. F., \& van der Werf, P. P. 2011, A\&A, 525, A119

Melnick, G. J., Tolls, V., Neufeld, D. A., et al. 2010, A\&A, 521, L27

Morris, M., \& Serabyn, E. 1996, ARA\&A, 34, 645

Müller, H. S. P., Thorwirth, S., Roth, D. A., \& Winnewisser, G. 2001, A\&A, 370, L49

Müller, H. S. P., Schlöder, F., Stutzki, J., \& Winnewisser, G. 2005, J. Mol. Struct., 742,215

Murray, N., Quataert, E., \& Thompson, T. A. 2005, ApJ, 618, 569

Neff, S. G., Ulvestad, J. S., \& Teng, S. H. 2004, ApJ, 611, 186

Neufeld, D. A., Lepp, S., \& Melnick, G. J. 1995, ApJS, 100, 132

Nordgren, T. E., Chengalur, J. N., Salpeter, E. E., \& Terzian, Y. 1997, AJ, 114, 77

Offer, A. R., van Hemert, M. C., \& van Dishoeck, E. F. 1994, J. Chem. Phys 100,362

Omont, A., Neri, R., Cox, P., et al. 2011, A\&A, 530, L3

Omont, A., Yang, C., Cox, P., et al. 2013, A\&A, 551, A115

Ott, S. 2010, in Astronomical Data Analysis Software and Systems XIX, eds

Y. Mizumoto, K.-I. Morita, \& M. Ohishi, ASP Conf. Ser., 434, 139

Pérez-Torres, M. A., Romero-Cañizales, C., Alberdi, A., \& Polatidis, A. 2009, A\&A, 507, L17
Pérez-Torres, M. A., Alberdi, A., Romero-Cañizales, C., \& Bondi, M. 2010, A\&A, 519, L5

Pickett, H. M., Poynter, R. L., Cohen, E. A., et al. 1998, J. Quant. Spectr. Rad. Transf., 60, 883

Pilbratt, G. L., Riedinger, J. R., Passvogel, T., et al. 2010, A\&A, 518, L1

Poglitsch, A., Waelkens, C., Geis, N., et al. 2010, A\&A, 518, L2

Prantzos, N., Aubert, O., \& Audouze, J. 1996, A\&A, 309, 760

Ptak, A., Hornschemeier, A., Zezas, A., et al. 2015, ApJ, 800, 104

Rolffs, R., Schilke, P., Wyrowski, F., et al. 2011, A\&A, 529, A76

Rosenberg, M. J. F., Meijerink, R., Israel, F. P., et al. 2014, A\&A, 568, A90

Sakamoto, K., Aalto, S., Costagliola, F., et al. 2013, ApJ, 764, 42

Sakamoto, K., Aalto, S., Combes, F., Evans, A., \& Peck, A. 2014, ApJ, 797, 90

Sanders, D. B., \& Mirabel, I. F. 1996, ARA\&A, 34, 749

Sanders, D. B., Mazzarella, J. M., Kim, D.-C., Surace, J. A., \& Soifer, B. T. 2003, AJ, 126, 1607

Sargent, A., \& Scoville, N. 1991, ApJ, 366, L1

Scoville, N. Z., \& Good, J. C. 1989, ApJ, 339, 149

Spoon, H. W. W., Farrah, D., Lebouteiller, V., et al. 2013, ApJ, 775, 127

Sturm, E., González-Alfonso, E., Veilleux, S., et al. 2011, ApJ, 733, L16

Tarchi, A., Castangia, P., Henkel, C., \& Menten, K. M. 2007, New A Rev., 51, 67

Tarchi, A., Castangia, P., Henkel, C., Surcis, G., \& Menten, K. M. 2011, A\&A, 525, A91

Telesco, C. M., Decher, R., \& Gatley, I. 1985, ApJ, 299, 896

Thompson, T. A., Quataert, E., \& Murray, N. 2005, ApJ, 630, 167

Ulvestad, J. S. 2009, AJ, 138, 1529

Valtchanov, I., Hopwood, R., Polehampton, E., et al. 2014, Exper. Astron., 37, 207

van der Werf, P. P., Isaak, K. G., Meijerink, R., et al. 2010, A\&A, 518, L42 van der Werf, P. P., Berciano Alba, A., Spaans, M., et al. 2011, ApJ, 741, L38 van Dishoeck, E. F., Herbst, E., \& Neufeld, D. A. 2013, Chem. Rev., 113, 9043 Veilleux, S., Meléndez, M., Sturm, E., et al. 2013, ApJ, 776, 27

Wilson, C. D., Petitpas, G. R., Iono, D., et al. 2008, ApJS, 178, 189

Wright, C. M., van Dishoeck, E. F., Black, J. H., et al. 2000, A\&A, 358, 689

Yang, C., Gao, Y., Omont, A., et al. 2013, ApJ, 771, L24

Zezas, A., Ward, M. J., \& Murray, S. S. 2003, ApJ, 594, L31 